\title{
Electronic structures of iMAX phases and their two-dimensional derivatives: A family of piezoelectric materials
}

\author{
Mohammad Khazaei, ${ }^{1, *}$ Vei Wang ${ }^{\dagger},{ }^{2}$ Cem Sevik, ${ }^{3}$ Ahmad Ranjbar, ${ }^{1}$ Masao Arai, ${ }^{4}$ and Seiji Yunoki ${ }^{1,5,6}$ \\ ${ }^{1}$ Computational Materials Science Research Team, \\ RIKEN Center for Computational Science (RCCS), Kobe, Hyogo 650-0047, Japan \\ ${ }^{2}$ Department of Applied Physics, Xi'an University of Technology, Xi'an 710054, China \\ ${ }^{3}$ Department of Mechanical Engineering, Anadolu University, Eskisehir 26555, Turkey \\ ${ }^{4}$ International Center for Materials Nanoarchitectonics, \\ National Institute for Materials Science (NIMS), 1-1 Namiki, Tsukuba 305-0044, Ibaraki, Japan \\ ${ }^{5}$ Computational Condensed Matter Physics Laboratory, RIKEN, Wako, Saitama 351-0198, Japan \\ ${ }^{6}$ Computational Quantum Matter Research Team, \\ RIKEN Center for Emergent Matter Science (CEMS), Wako, Saitama 351-0198, Japan
}

(Dated: June 26, 2018)

\begin{abstract}
Recently, a group of MAX phases, $\left(\mathrm{Mo}_{2 / 3} \mathrm{Y}_{1 / 3}\right)_{2} \mathrm{AlC},\left(\mathrm{Mo}_{2 / 3} \mathrm{Sc}_{1 / 3}\right)_{2} \mathrm{AlC}$, $\left(\mathrm{W}_{2 / 3} \mathrm{Sc}_{1 / 3}\right)_{2} \mathrm{AlC}$, $\left(\mathrm{W}_{2 / 3} \mathrm{Y}_{1 / 3}\right)_{2} \mathrm{AlC}$, and $\left(\mathrm{V}_{2 / 3} \mathrm{Zr}_{1 / 3}\right)_{2} \mathrm{AlC}$, with in-plane ordered double transition metals, named iMAX phases, have been synthesized. Experimentally, some of these MAX phases can be chemically exfoliated into two-dimensional (2D) single- or multilayered transition metal carbides, so-called MXenes. Accordingly, the 2D nanostructures derived from iMAX phases are named iMXenes. Here, we investigate the structural stabilities and electronic structures of the experimentally discovered iMAX phases and their possible iMXene derivatives. We show that the iMAX phases and their pristine, F, or OH-terminated iMXenes are metallic. However, upon $\mathrm{O}$ termination, $\left(\mathrm{Mo}_{2 / 3} \mathrm{Y}_{1 / 3}\right)_{2} \mathrm{C}$, $\left(\mathrm{Mo}_{2 / 3} \mathrm{Sc}_{1 / 3}\right)_{2} \mathrm{C}$, $\left(\mathrm{W}_{2 / 3} \mathrm{Y}_{1 / 3}\right)_{2} \mathrm{C}$, and $\left(\mathrm{W}_{2 / 3} \mathrm{Sc}_{1 / 3}\right)_{2} \mathrm{C}$ iMXenes turn into semiconductors. The semiconducting iMXenes may find applications in piezoelectricity owing to the absence of centrosymmetry. Our calculations reveal that the semiconducting iMXenes possess giant piezoelectric coefficients as large as $45 \times 10^{-10} \mathrm{C} / \mathrm{m}$.
\end{abstract}

PACS numbers: 81.05.Je, 62.20.Dc, 62.20.-x, 71.20.-b

\section{INTRODUCTION}

Over the past couple of years, tremendous improvements have been achieved in the synthesis of crystalline MAX phases - a large family of transition metal carbides and nitrides with layered hexagonal structures and a chemical formula of $\mathrm{M}_{n+1} \mathrm{AX}_{n}$, where " $\mathrm{M}$ " is an early transition metal ( $\mathrm{Sc}, \mathrm{Ti}, \mathrm{V}, \mathrm{Nb}, \mathrm{Ta}, \mathrm{Cr}$, and Mo), "A" belongs to groups XIII-XVI in the periodic table ( $\mathrm{Al}$, Ga, In, Tl, Si, Ge, Sn, P, As, Bi, and S), "X" stands for $\mathrm{C}$ or $\mathrm{N}$, and $n=1-3$ [1-5]. Traditionally, MAX phases are well-known materials because of their ceramic nature with a high structural stability against fatigue, creep, thermal shock, and corrosive reactions; therefore, they have been adopted to many structural applications $[1,2]$. In recent experiments, it has been shown that the "A" element can be removed from some MAX phases by using combinations of an appropriate acid treatment and sonication. This process results in the formation of single or multilayered two-dimensional (2D) transition metal carbides or nitrides, so-called MXenes [6, 7]. During the exfoliation process, depending on the type of acid solution (e.g., HF acid), the surfaces of MXenes are usually terminated with a mixture of $\mathrm{F}, \mathrm{OH}$, and $\mathrm{O}$ groups [6-

${ }^{\dagger}$ V.W. and C.S. contributed equally to this work.

*Electronic address: khazaei@riken.jp
8]. As stated in recent reviews covering the current status of research and development on this novel family of low-dimensional systems $[9,10]$, MXenes may have potential electronic [11-20], magnetic [11, 21-27], thermoelectric [11, 12], optoelectronic [28], ferroelectric [29], superconducting [30, 31], and energy storage applications [32-34], as well as applications in energy conversion devices [35-38] and in developing new composite materials $[39,40]$. Hence, interest in the synthesis of novel MAX phases and 2D MXenes has rapidly increased.

One of the unique structural characteristics of MAX phases is that they can be formed in various solid solutions with pure or mixtures of $\mathrm{M}, \mathrm{A}$, and $\mathrm{X}$ elements [41-51]. In addition to traditional $\mathrm{M}_{n+1} \mathrm{AX}_{n} \mathrm{MAX}$ phases, several sets of crystalline ordered double transition metal MAX phases, $\mathrm{M}_{2}^{\prime} \mathrm{M}^{\prime \prime} \mathrm{AX}_{2}, \mathrm{M}_{2}^{\prime} \mathrm{M}_{2}^{\prime \prime} \mathrm{AX}_{3}$ [52, 53], and $\left(\mathrm{M}_{2 / 3}^{\prime} \mathrm{M}_{1 / 3}^{\prime \prime}\right)_{2} \mathrm{AX}$ [54-56], have been discovered recently. In $\mathrm{M}_{2}^{\prime} \mathrm{M}^{\prime \prime} \mathrm{AX}_{2}$ and $\mathrm{M}_{2}^{\prime} \mathrm{M}_{2}^{\prime \prime} \mathrm{AX}_{3}$, the layers are occupied purely with an $\mathrm{M}^{\prime}, \mathrm{M}^{\prime \prime}$, $\mathrm{A}$, or X element. In $\left(\mathrm{M}_{2 / 3}^{\prime} \mathrm{M}_{1 / 3}^{\prime \prime}\right)_{2} \mathrm{AX}$, however, the double transition metals of $\mathrm{M}^{\prime}$ and $\mathrm{M}^{\prime \prime}$ are ordered in-plane, as shown in Fig. 1(a). Theoretically, another set of MAX phases with double transition metals $\left(\mathrm{M}_{1 / 2}^{\prime} \mathrm{M}_{1 / 2}^{\prime \prime}\right)_{n+1} \mathrm{AX}_{n}$ with a high ordering tendency has also been predicted, in which each layer is occupied purely with one type of element $[49,50]$. In fact, the current experimental attempts to synthesize the latter MAX phase are quite close to becoming successful [57]. These developments clearly demonstrate that many partially or perfectly ordered crystalline MXenes 
(a)

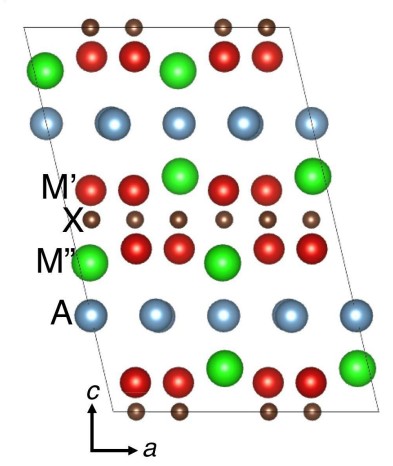

(c)
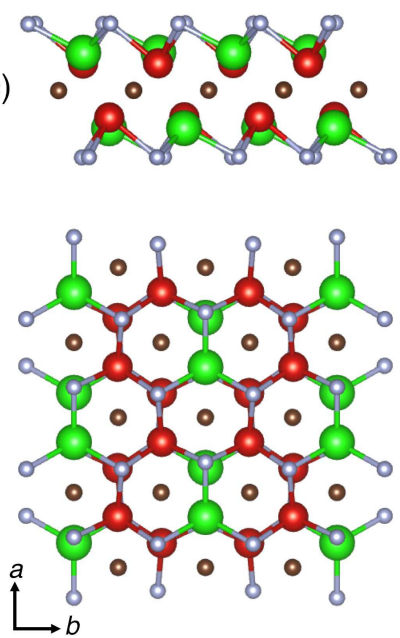

(b)

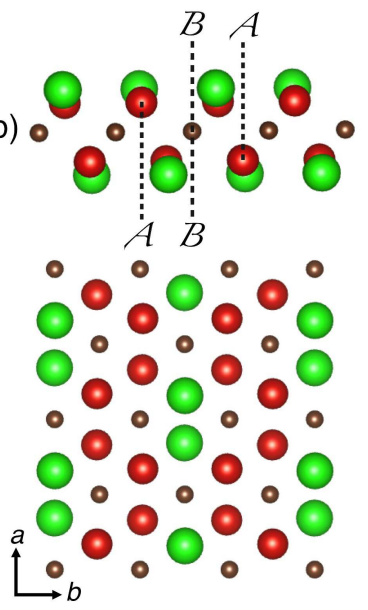

(d)
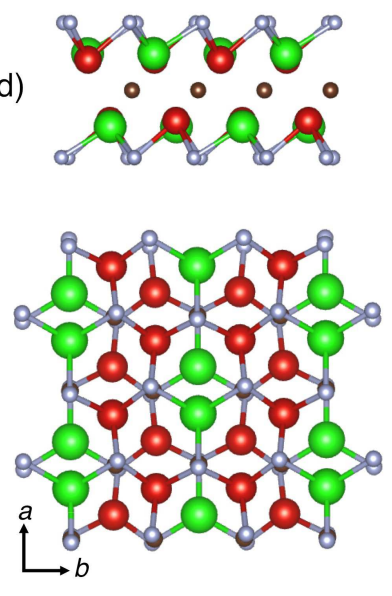

and $\left(\mathrm{V}_{2 / 3} \mathrm{Zr}_{1 / 3}\right)_{2} \mathrm{AlC}$ and their possible $2 \mathrm{D}$ iMXene derivatives, $\left(\mathrm{Mo}_{2 / 3} \mathrm{Sc}_{1 / 3}\right)_{2} \mathrm{C}, \quad\left(\mathrm{Mo}_{2 / 3} \mathrm{Y}_{1 / 3}\right)_{2} \mathrm{C}$, $\left(\mathrm{W}_{2 / 3} \mathrm{Sc}_{1 / 3}\right)_{2} \mathrm{C},\left(\mathrm{W}_{2 / 3} \mathrm{Y}_{1 / 3}\right)_{2} \mathrm{C}$, and $\left(\mathrm{V}_{2 / 3} \mathrm{Zr}_{1 / 3}\right)_{2} \mathrm{C}$ [for the crystal structure, see Fig. 1(b)]. All of the considered iMAX phases are found to be metallic, in accordance with a previous theoretical study [59]. Single-layered iMXenes without functionalization are also found to be metallic. However, we find that the single-layered $\left(\mathrm{Mo}_{2 / 3} \mathrm{Sc}_{1 / 3}\right)_{2} \mathrm{C}, \quad\left(\mathrm{Mo}_{2 / 3} \mathrm{Y}_{1 / 3}\right)_{2} \mathrm{C}, \quad\left(\mathrm{W}_{2 / 3} \mathrm{Sc}_{1 / 3}\right)_{2} \mathrm{C}$, and $\left(\mathrm{W}_{2 / 3} \mathrm{Y}_{1 / 3}\right)_{2} \mathrm{C}$ iMXenes functionalized with $\mathrm{O}$ are semiconductors with indirect band gaps. Moreover, we predict that these iMXenes exhibit giant piezoelectricity with a piezoelectric coefficient of $\sim 45 \times 10^{-10} \mathrm{C} / \mathrm{m}$. Therefore, the O-functionalized iMXenes are promising for exceptional piezoelectric applications.

The rest of this paper is organized as follows. The calculation method is briefly described in Sec. II. The electronic properties of the iMAX phases are reported in Sec. III A, and the structural stability of iMXenes and their electronic, mechanical, and piezoelectric properties are examined in Sec. III B. Finally, the paper is concluded in Sec. IV.

\section{CALCULATION METHOD}

First-principles calculations based on densityfunctional theory are performed using the VASP code [60]. The exchange-correlation energies are taken into account within the generalized gradient approximation (GGA) via Perdew-Burke - Ernzerhof functional [61]. The wave functions are constructed by the projected augmented wave method with a plane wave cutoff energy of $700 \mathrm{eV}$. For optimizing the atomic structure of the conventional unit cell of iMAX phases [see Fig. 1(a)], at least $6 \times 12 \times 4$ Monkhorst Pack $k$ points [62] are considered to integrate the Brillouin zone. The mechanical properties of the derived iMXenes are calculated using $14 \times 10 \times 1 k$ points. The energy and residual force convergence criteria are set to be $10^{-8} \mathrm{eV} /$ cell and $0.0005 \mathrm{eV} / \AA$, respectively. In order to avoid any interaction between the single layer with its images along the $c$ axis, we have used a vacuum space of more than $22 \AA$. Because the multilayered structures of iMXenes is not considered, we do not apply any special treatment for the van der Waals interactions. The phonon calculations are performed by employing the finite displacement method. The displaced atomic configurations are obtained using the PHONOPY package [63].

The adsorption energy $E_{\text {a }}$ per termination group $\mathrm{T}$ $(\mathrm{T}=\mathrm{F}, \mathrm{O}$, and $\mathrm{OH})$ is defined as $E_{\mathrm{a}}=\left(E_{\mathrm{tot}}-E_{\mathrm{p}}-\right.$ $\left.2 E_{\mathrm{T}}\right) / 2$, where $E_{\text {tot }}, E_{\mathrm{p}}$, and $E_{\mathrm{T}}$ are the total energies of the functionalized iMXene, the pristine iMXene, and a termination group, respectively. $E_{\mathrm{T}}$ is evaluated from the total energies of stable $\mathrm{O}_{2}, \mathrm{H}_{2}$, and $\mathrm{F}_{2}$ molecules.

In order to examine the mechanical properties of pristine and functionalized iMXenes, the elastic strain en- 
TABLE I: Experimental and theoretical lattice parameters for experimentally synthesized iMAX phases in a base-centered monoclinic cell with $C 2 / c$ symmetry and thus $\alpha=\gamma=90^{\circ}$ (see Fig. 1).

\begin{tabular}{llllll}
\hline \hline iMAX phase & $a(\AA)$ & $b(\AA)$ & $c(\AA)$ & $\beta\left(^{\circ}\right)$ \\
\hline$\left(\mathrm{Mo}_{2 / 3} \mathrm{Sc}_{1 / 3}\right)_{2} \mathrm{AlC}$ & & & & \\
Experiment [54] & 9.335 & 5.391 & 13.861 & 103.191 \\
This study & 9.349 & 5.417 & 13.925 & 103.558 \\
$\left(\mathrm{Mo}_{2 / 3} \mathrm{Y}_{1 / 3}\right)_{2} \mathrm{AlC}$ & & & & \\
Experiment [55] & 9.679 & 5.285 & 14.076 & 103.359 \\
This study & 9.569 & 5.540 & 14.126 & 103.655 \\
$\left(\mathrm{~W}_{2 / 3} \mathrm{Sc}_{1 / 3}\right)_{2} \mathrm{AlC}$ & & & & \\
Experiment [56] & 9.368 & 5.404 & 13.960 & - \\
This study & 9.323 & 5.395 & 13.976 & 103.441 \\
$\left(\mathrm{~W}_{2 / 3} \mathrm{Y}_{1 / 3}\right)_{2} \mathrm{AlC}$ & & & & \\
Experiment [56] & 9.510 & 5.490 & 14.220 & - \\
This study & 9.551 & 5.524 & 14.136 & 103.617 \\
$\left(\mathrm{~V}_{2 / 3} \mathrm{Zr}_{1 / 3}\right)_{2} \mathrm{AlC}$ & & & & \\
Experiment [55] & 9.172 & 5.281 & 13.642 & 103.071 \\
This study & 9.160 & 5.275 & 13.617 & 103.069 \\
\hline \hline
\end{tabular}

ergy per unit area is first calculated by applying in-plane strains in various directions in the range $-2 \% \leq \varepsilon \leq 2 \%$ with an increment of $0.5 \%$, where $\varepsilon=\frac{a-a_{0}}{a_{0}} \times 100 \%$ and $a_{0}$ and $a$ are the lattice parameters before and after applying the strain, respectively. Then, the elastic constant $C_{i j}$ is obtained by fitting the change in the total energy versus the applied strain with a second-order polynomial. This is carried out using the VASPKIT code [64]. Note that, following the standard Voigt notation [65], the elastic strain energy $E_{\mathrm{s}}$ per unit area can be expressed as $E_{\mathrm{s}}=\frac{1}{2} C_{11} \varepsilon_{x x}^{2}+\frac{1}{2} C_{22} \varepsilon_{y y}^{2}+\frac{1}{2} C_{12} \varepsilon_{x x} \varepsilon_{y y}+2 C_{66} \varepsilon_{x y}^{2}$ for a $2 \mathrm{D}$ system, where $\varepsilon_{x x}, \varepsilon_{y y}$, and $\varepsilon_{x y}$ are tensile strains with $x$ and $y$ directions that are chosen along the $a$ and $b$ lattice vectors, respectively [66]. The coefficient of the piezoelectric tensor, $e_{i j}$, is calculated on the basis of density-functional perturbation theory (DFPT) [67], as implemented in the VASP code. Because of the crystal symmetry studied here, $e_{11}$ and $e_{12}$ are the only independent piezoelectric coefficients. The $i j$ component of the piezoelectric strain tensor $d_{i j}[68]$ is finally obtained as

$$
d_{11}=\frac{e_{11} C_{22}-e_{12} C_{12}}{C_{11} C_{22}-C_{12}^{2}}
$$

and

$$
d_{12}=\frac{e_{12} C_{11}-e_{11} C_{12}}{C_{11} C_{22}-C_{12}^{2}}
$$

\section{RESULTS AND DISCUSSION}

\section{A. iMAX phases}

The iMAX phase possesses a base-centered monoclinic structure with $C 2 / c$ symmetry. The calculated lattice parameters of five iMAX phases, $\left(\mathrm{Mo}_{2 / 3} \mathrm{Sc}_{1 / 3}\right)_{2} \mathrm{AlC}$, $\left(\mathrm{Mo}_{2 / 3} \mathrm{Y}_{1 / 3}\right)_{2} \mathrm{AlC},\left(\mathrm{W}_{2 / 3} \mathrm{Sc}_{1 / 3}\right)_{2} \mathrm{AlC},\left(\mathrm{W}_{2 / 3} \mathrm{Y}_{1 / 3}\right)_{2} \mathrm{AlC}$, and $\left(\mathrm{V}_{2 / 3} \mathrm{Zr}_{1 / 3}\right)_{2} \mathrm{AlC}$, are summarized in Table I. These results are in excellent agreement with the available experimental data $[54,55]$. Note that, following the experimental reports, the lattice parameters in Table I are for the conventional cell, which is two times larger than the primitive cell.

In order to check the dynamic stabilities of these iMAX phases, the vibrational properties are investigated. Figure 2 shows the calculated phonon dispersion and projected density of states for the conventional cell of $\left(\mathrm{Mo}_{2 / 3} \mathrm{Y}_{1 / 3}\right)_{2} \mathrm{AlC}$ as an example. The phonon results for the other iMAX phases are also provided in the Supplemental Material [69]. None of the considered iMAX phases have a negative phonon frequency, indicating their structural stability at zero temperature. The projected phonon density of states clearly suggests that phonon modes related to the $\mathrm{M}^{\prime}-\mathrm{C}$ or $\mathrm{M}^{\prime \prime}-\mathrm{C}$ vibrations appear above $12 \mathrm{THz}$ and have higher frequencies than the phonon modes related to the $\mathrm{M}^{\prime}-\mathrm{M}^{\prime}, \mathrm{M}^{\prime}-\mathrm{M}^{\prime \prime}, \mathrm{M}^{\prime}-\mathrm{Al}$, $\mathrm{M}^{\prime \prime}-\mathrm{Al}$, or $\mathrm{Al}-\mathrm{Al}$ vibrations [59]. This implies that, in analogy to the other members of the MAX phase family, the force constants of the $\mathrm{M}^{\prime}-\mathrm{C}$ or $\mathrm{M}^{\prime \prime}-\mathrm{C}$ bonds are higher than those of the other bonds [70]. In other words, the $\mathrm{M}^{\prime}-\mathrm{C}$ or $\mathrm{M}^{\prime \prime}-\mathrm{C}$ bonds are stronger than the $\mathrm{M}^{\prime}-\mathrm{M}^{\prime}$, $\mathrm{M}^{\prime}-\mathrm{M}^{\prime \prime}$, or $\mathrm{Al}-\mathrm{Al}$ bonds. If the bonding among $\mathrm{M}^{\prime}, \mathrm{M}^{\prime \prime}$, and $\mathrm{C}$ is stronger than that with $\mathrm{Al}$, there is a higher chance for the successful exfoliation of iMAX phases into iMXenes [70]. In fact, currently, $\left(\mathrm{Mo}_{2 / 3} \mathrm{Sc}_{1 / 3}\right)_{2} \mathrm{AlC}$ and $\left(\mathrm{Mo}_{2 / 3} \mathrm{Y}_{1 / 3}\right)_{2} \mathrm{AlC}$ have been exfoliated to $\mathrm{Mo}_{1.33} \mathrm{C}$ experimentally $[54,71]$. Likewise, $\left(\mathrm{W}_{2 / 3} \mathrm{Sc}_{1 / 3}\right)_{2} \mathrm{AlC}$ and $\left(\mathrm{W}_{2 / 3} \mathrm{Y}_{1 / 3}\right)_{2} \mathrm{AlC}$ have been exfoliated to $\mathrm{W}_{1.33} \mathrm{C}$ experimentally [56].

We also examine the electronic structures of $\left(\mathrm{Mo}_{2 / 3} \mathrm{Sc}_{1 / 3}\right)_{2} \mathrm{AlC},\left(\mathrm{Mo}_{2 / 3} \mathrm{Y}_{1 / 3}\right)_{2} \mathrm{AlC},\left(\mathrm{W}_{2 / 3} \mathrm{Sc}_{1 / 3}\right)_{2} \mathrm{AlC}$, $\left(\mathrm{W}_{2 / 3} \mathrm{Y}_{1 / 3}\right)_{2} \mathrm{AlC}$, and $\left(\mathrm{V}_{2 / 3} \mathrm{Zr}_{1 / 3}\right)_{2} \mathrm{AlC}$. For instance, the results for $\left(\mathrm{Mo}_{2 / 3} \mathrm{Y}_{1 / 3}\right)_{2} \mathrm{AlC}$ are shown in Fig. 3. The band structures and density of states clearly show that, similar to the other MAX phases, the iMAX phases are metallic. This can be simply explained by considering that in MAX phases, including iMAX phases, the transition metal atoms are connected in each layer, resulting in the dominant contribution of the $d$ orbitals of the transition metals to the states near the Fermi energy and consequently to the electrical conductivity of these phases. The projected DOSs of the $\mathrm{M}^{\prime}$ and $\mathrm{M}^{\prime \prime}$ transition metals are similarly distributed around the Fermi energy. However, the $\mathrm{M}^{\prime}$ atoms have larger contribution since, in $\left(\mathrm{M}_{2 / 3}^{\prime} \mathrm{M}_{1 / 3}^{\prime \prime}\right)_{2} \mathrm{AX}$, the number of $\mathrm{M}^{\prime}$ atoms is larger than that of $\mathrm{M}^{\prime \prime}$ atoms.

The projected density of states (PDOS) in Fig. 3 also 


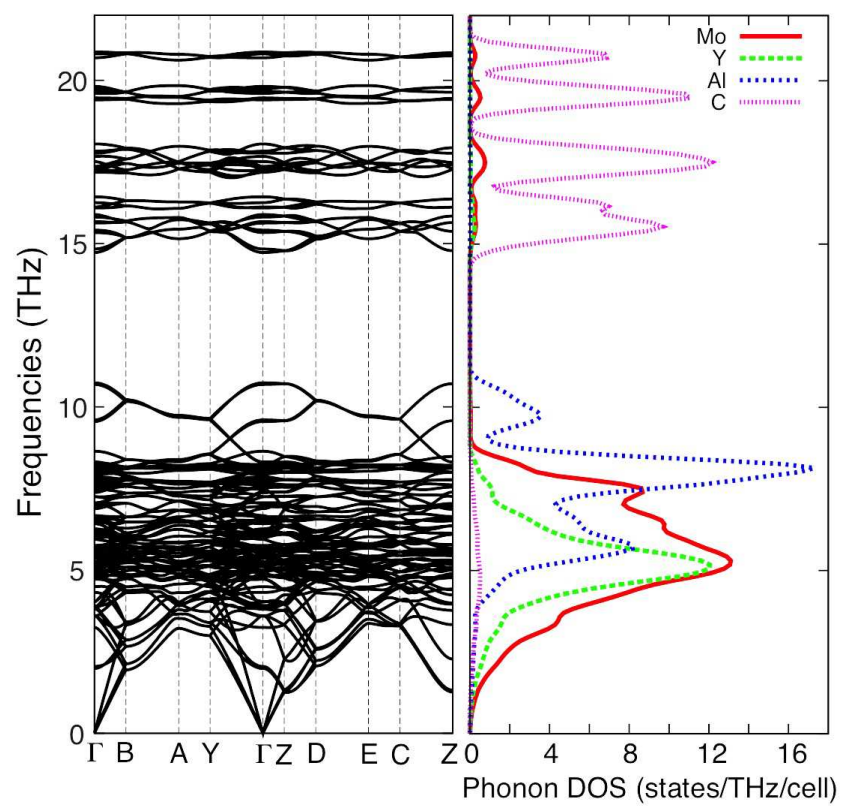

FIG. 2: Phonon spectrum and phonon density of states (DOS) for $\left(\mathrm{Mo}_{2 / 3} \mathrm{Y}_{1 / 3}\right)_{2} \mathrm{AlC} . \Gamma(0,0,0), \mathrm{B}(-0.5,0,0), \mathrm{A}(-0.5,0.5,0)$, $\mathrm{Y}(0,0.5,0), \mathrm{Z}(0,0,0.5), \mathrm{D}(-0.5,0,0.5), \mathrm{E}(-0.5,0.5,0.5)$, and $\mathrm{C}(0,0.5,0.5)$ are high symmetric points of the Brillouin zone.

shows that there is significant hybridization between the $\mathrm{C}$ atoms and the transition metals $\mathrm{M}^{\prime}$ and $\mathrm{M}^{\prime \prime}$. The states between -6 and $-3 \mathrm{eV}(-12$ and $-10 \mathrm{eV})$ result from the hybridization between the $p(s)$ orbitals of $\mathrm{C}$ and the $d$ orbitals of $\mathrm{M}^{\prime}$ and $\mathrm{M}^{\prime \prime}$. There also exists hybridization between the $p$ orbitals of $\mathrm{Al}$ and the $d$ orbitals of $\mathrm{M}^{\prime}$ and $\mathrm{M}^{\prime \prime}$, appearing in a broad range of energies between -6 and $-1 \mathrm{eV}$. However, this hybridization is not as strong as the hybridization between the atomic orbitals of $\mathrm{C}$ and $\mathrm{M}^{\prime}$ or $\mathrm{M}^{\prime \prime}$.

In both the MAX and iMAX phases, the energy gaps at around $-9 \mathrm{eV}$ separate the valence bands from other low lying states such as the $\mathrm{M}$ or $\mathrm{C} s$ bands. Just above these energy gaps, the states at the lowest part of the valence bands result from the hybridization between the atomic orbitals of $\mathrm{Al}$ atoms in the $\mathrm{Al}-\mathrm{Al}$ bonds (see the Supplemental Material [69]). In typical MAX phases where the $\mathrm{Al}$ atoms are located on a uniform hexagonal lattice, the energy bands in this energy region show a parabolic dispersion, and the total DOSs do not show notable features. In the iMAX phases, on the other hand, additional features appear in the energy bands and DOSs. For example, the total DOS becomes almost zero around -7 $\mathrm{eV}$ for $\left(\mathrm{Mo}_{2 / 3} \mathrm{Y}_{1 / 3}\right) \mathrm{AlC}$ (see Fig. 3). Such a pseudogap originates from the Kagome-like alignment of $\mathrm{Al}$ atoms. Thus, these features can be considered as a kind of fingerprint of the iMAX phases. In addition, the $\mathrm{Al}-\mathrm{Al}$ bond lengths in the $\left(\mathrm{M}_{2 / 3}^{\prime} \mathrm{M}_{1 / 3}^{\prime \prime}\right)_{2} \mathrm{AC}$ iMAX phases are slightly shorter than those in $\mathrm{M}_{2}^{\prime} \mathrm{AC}$ or $\mathrm{M}_{2}^{\prime \prime} \mathrm{AC}$; therefore, we expect that the $\mathrm{Al}-\mathrm{Al}$ bonds in the iMAX phases should be slightly stronger than those in other MAX phases.
Recently, we have performed a very intensive study on the possibility of exfoliation of 82 different MAXphase compounds into 2D MXenes [70]. According to our analysis, MAX phases with exfoliation energies less than $0.205 \mathrm{eV} / \AA^{2}$ have a very high chance to be exfoliated into 2D MXenes. We have performed a similar analysis of the exfoliation energies of iMAX phases into 2D iMXenes. The exfoliation energies of $\left(\mathrm{Mo}_{2 / 3} \mathrm{Sc}_{1 / 3}\right)_{2} \mathrm{AlC},\left(\mathrm{Mo}_{2 / 3} \mathrm{Y}_{1 / 3}\right)_{2} \mathrm{AlC},\left(\mathrm{W}_{2 / 3} \mathrm{Sc}_{1 / 3}\right)_{2} \mathrm{AlC}$, $\left(\mathrm{W}_{2 / 3} \mathrm{Y}_{1 / 3}\right)_{2} \mathrm{AlC}$, and $\left(\mathrm{V}_{2 / 3} \mathrm{Zr}_{1 / 3}\right)_{2} \mathrm{AlC}$ to their corresponding iMXenes, $\left(\mathrm{Mo}_{2 / 3} \mathrm{Sc}_{1 / 3}\right)_{2} \mathrm{C},\left(\mathrm{Mo}_{2 / 3} \mathrm{Y}_{1 / 3}\right)_{2} \mathrm{C}$, $\left(\mathrm{W}_{2 / 3} \mathrm{Sc}_{1 / 3}\right)_{2} \mathrm{C}$, $\left(\mathrm{W}_{2 / 3} \mathrm{Y}_{1 / 3}\right)_{2} \mathrm{C}$, and $\left(\mathrm{V}_{2 / 3} \mathrm{Zr}_{1 / 3}\right)_{2} \mathrm{C}$, are estimated to be around $0.156,0.172,0.177,0.162$, and $0.185 \mathrm{eV} / \AA^{2}$, respectively. Since all obtained exfoliation energies are less than $0.205 \mathrm{eV} / \AA^{2}$, we predict, according to our previous analysis [70], that the above iMAX phases can be exfoliated into 2D iMXenes, as indeed observed in experiments $[54,56,71]$.

\section{B. iMXenes}

Upon chemical exfoliation, the MAX phases can be exfoliated into single- or multilayered MXenes, and their surfaces are terminated with mixture of $\mathrm{F}, \mathrm{OH}$, and $\mathrm{O}$ groups [6-8] owing to the reactivity of the surface transition metals. Theoretical studies indicate that fully saturated MXenes are thermodynamically more favorable than partially saturated ones $[11,72]$. Thermodynamic simulations show that, by proper control of the chemical potential, the surfaces of MXenes can be saturated with a particular type of chemical group [11, 72]. Although the synthesis of MXenes with a particular surface termination is still a challenge in wet chemistry experiments, there have been developments regarding the control of the surface termination of MXenes by using a thermal process [8]. Using this technique, it might be possible to synthesize pristine MXenes. Therefore, we theoretically study the structural stability of iMXenes and their electronic properties.

\section{Atomic structure and dynamic stability}

It has been repeatedly shown that exfoliated MXenes possess many interesting properties and applications that their original MAX phases do not show [9, 10]. For instance, although all MAX phases are metallic, upon exfoliation and proper surface functionalization, some of them turn into semiconductors [11] or topological insulators $[14,17,19,73-75]$. Such interesting properties and applications motivate us to investigate the electronic properties of iMXenes in detail.

The electronic structures of MXenes are significantly affected by the type and position of the chemical groups absorbed on the surfaces of MXenes [11]. Hence, in order to propose the lowest energy atomic configuration for functionalized iMXenes, it is necessary to investigate 


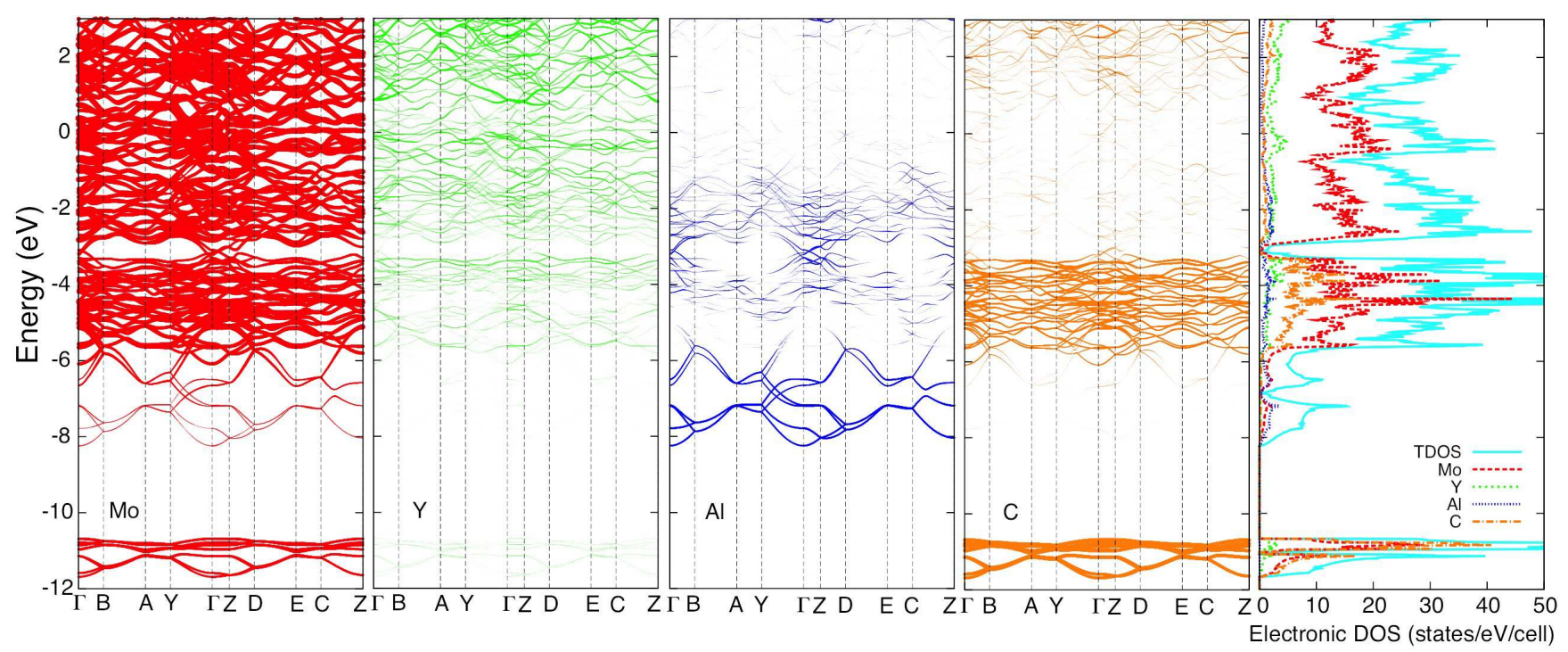

FIG. 3: Band structure and density of states (DOS) of $\left(\mathrm{Mo}_{2 / 3} \mathrm{Y}_{1 / 3}\right)_{2} \mathrm{AlC}$, projected onto the compositional elements, Mo, Y, $\mathrm{Al}$, and C. TDOS stands for total density of states. The Fermi energy is set at zero.
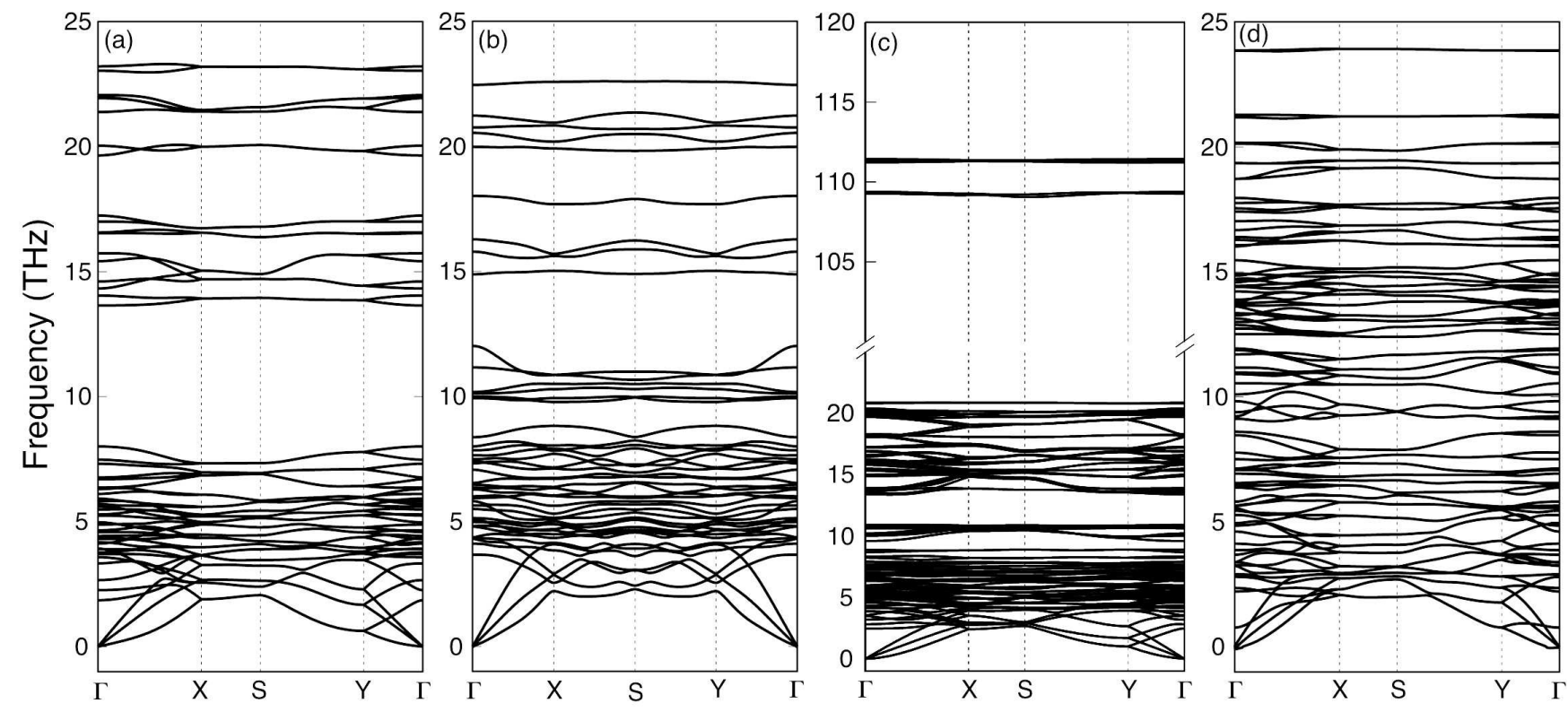

FIG. 4: Phonon band structures of (a) pristine $\left(\mathrm{Mo}_{2 / 3} \mathrm{Y}_{1 / 3}\right)_{2} \mathrm{C}$, (b) $\left(\mathrm{Mo}_{2 / 3} \mathrm{Y}_{1 / 3}\right)_{2} \mathrm{CF}_{2}$, (c) $\left(\mathrm{Mo}_{2 / 3} \mathrm{Y}_{1 / 3}\right)_{2} \mathrm{C}(\mathrm{OH})_{2}$, and (d) $\left(\mathrm{Mo}_{2 / 3} \mathrm{Y}_{1 / 3}\right)_{2} \mathrm{CO}_{2}$ iMXenes. $\Gamma(0,0,0), \mathrm{X}(0.5,0,0), \mathrm{S}(0.5,0.5,0)$, and $\mathrm{Y}(0,0.5,0)$ are high symmetric points of the Brillouin zone.

the stability of various iMXenes with functionalization at different adsorption sites. Although the atomic configurations of the $\mathrm{Al}$ atoms in typical MAX phases (with $\mathrm{Al}$ atoms forming a hexagonal lattice) and in the iMAX phases (with $\mathrm{Al}$ atoms forming a Kagome-like lattice) are different, the pristine iMXene $\left(\mathrm{M}_{2 / 3}^{\prime} \mathrm{M}_{1 / 3}^{\prime \prime}\right)_{2} \mathrm{C}$ after removing $\mathrm{Al}$ atoms possesses a surface structure similar to that of the pristine $\mathrm{M}_{2} \mathrm{C}$ MXene with two types of hollow sites, $\mathcal{A}$ and $\mathcal{B}$, as indicated in Fig. 1(b).

For hollow site $\mathcal{A}(\mathcal{B})$, there is no (there is a) $\mathrm{C}$ atom underneath it. Previous calculations have revealed that when the surfaces of MXenes are saturated with $\mathrm{F}, \mathrm{OH}$, or $\mathrm{O}$, these chemical groups prefer to be adsorbed at the hollow sites rather than adsorbed directly on top of the transition metals $[11,12]$. Therefore, depending on the positions of the adsorbed atoms at the hollow sites, there are three possibilities for the functionalized iMXenes, i.e., $\mathcal{A} \mathcal{A}, \mathcal{B B}$, and $\mathcal{A B}$ model structures. In model structure $\mathcal{A} \mathcal{A}$, the opposite sides of surfaces are saturated with chemical groups adsorbed at $\mathcal{A}$-type hollow sites [see Fig. 1(c)]. In model structure $\mathcal{B B}$, the opposite sides of surfaces are saturated with chemical groups ad- 
TABLE II: Total energy differences (eV per functional group) of different model structures $\mathcal{A} \mathcal{A}, \mathcal{A B}$, and $\mathcal{B B}$ for $\left(\mathrm{M}_{2 / 3}^{\prime} \mathrm{M}_{1 / 3}^{\prime \prime}\right)_{2} \mathrm{CT}_{2}$ with $\mathrm{T}=\mathrm{F}, \mathrm{OH}$, and $\mathrm{O}$. The total energy of the model structure with the lowest energy is set to be zero. $E_{\mathrm{a}}$ is the adsorption energy (eV per functional group).

\begin{tabular}{lcccc}
\hline \hline Functionalized iMXene & $\mathcal{A A}$ & $\mathcal{A B}$ & $\mathcal{B B}$ & $E_{\mathrm{a}}$ \\
\hline$\left(\mathrm{Mo}_{2 / 3} \mathrm{Sc}_{1 / 3}\right)_{2} \mathrm{CF}_{2}$ & 0.000 & 0.160 & 0.030 & -4.729 \\
$\left(\mathrm{Mo}_{2 / 3} \mathrm{Y}_{1 / 3}\right)_{2} \mathrm{CF}_{2}$ & 0.000 & 0.105 & 0.191 & -4.689 \\
$\left(\mathrm{~W}_{2 / 3} \mathrm{Sc}_{1 / 3}\right)_{2} \mathrm{CF}_{2}$ & 0.000 & 0.014 & 0.189 & -4.561 \\
$\left(\mathrm{~W}_{2 / 3} \mathrm{Y}_{1 / 3}\right)_{2} \mathrm{CF}_{2}$ & 0.000 & 0.062 & 0.061 & -4.530 \\
$\left(\mathrm{~V}_{2 / 3} \mathrm{Zr}_{1 / 3}\right)_{2} \mathrm{CF}_{2}$ & 0.000 & 0.014 & 0.318 & -4.982 \\
$\left(\mathrm{Mo}_{2 / 3} \mathrm{Sc}_{1 / 3}\right)_{2} \mathrm{CO}_{2}$ & 0.000 & 0.154 & 0.307 & -4.173 \\
$\left(\mathrm{Mo}_{2 / 3} \mathrm{Y}_{1 / 3}\right)_{2} \mathrm{CO}_{2}$ & 0.000 & 0.053 & 0.221 & -4.054 \\
$\left(\mathrm{~W}_{2 / 3} \mathrm{Sc}_{1 / 3}\right)_{2} \mathrm{CO}_{2}$ & 0.000 & 0.168 & 0.370 & -4.533 \\
$\left(\mathrm{~W}_{2 / 3} \mathrm{Y}_{1 / 3}\right)_{2} \mathrm{CO}_{2}$ & 0.000 & 0.142 & 0.324 & -4.433 \\
$\left(\mathrm{~V}_{2 / 3} \mathrm{Zr}_{1 / 3}\right)_{2} \mathrm{CO}_{2}$ & 0.000 & 0.193 & 0.430 & -4.982 \\
$\left(\mathrm{Mo}_{2 / 3} \mathrm{Sc}_{1 / 3}\right)_{2} \mathrm{C}(\mathrm{OH})_{2}$ & 0.000 & 0.1073 & 0.294 & -4.166 \\
$\left(\mathrm{Mo}_{2 / 3} \mathrm{Y}_{1 / 3}\right)_{2} \mathrm{C}(\mathrm{OH})_{2}$ & 0.000 & 0.048 & 0.332 & -4.074 \\
$\left(\mathrm{~W}_{2 / 3} \mathrm{Sc}_{1 / 3}\right)_{2} \mathrm{C}(\mathrm{OH})_{2}$ & 0.000 & 0.086 & 0.270 & -4.082 \\
$\left(\mathrm{~W}_{2 / 3} \mathrm{Y}_{1 / 3}\right)_{2} \mathrm{C}(\mathrm{OH})_{2}$ & 0.000 & 0.016 & 0.344 & -3.988 \\
$\left(\mathrm{~V}_{2 / 3} \mathrm{Zr}_{1 / 3}\right)_{2} \mathrm{C}(\mathrm{OH})_{2}$ & 0.000 & 0.169 & 0.219 & -4.363 \\
\hline \hline
\end{tabular}

sorbed at $\mathcal{B}$-type hollow sites [see Fig. 1(d)]. In model structure $\mathcal{A B}$, one side of the surface is saturated with chemical groups adsorbed at $\mathcal{A}$-type hollow sites, and the opposite side is saturated with chemical groups adsorbed at $\mathcal{B}$-type hollow sites. Note that each unit formula of $\left(\mathrm{M}_{2 / 3}^{\prime} \mathrm{M}_{1 / 3}^{\prime \prime}\right)_{2} \mathrm{C}$ requires two termination groups $(\mathrm{T}=\mathrm{F}, \mathrm{OH}$, and $\mathrm{O})$ for the full surface termination in $\left(\mathrm{M}_{2 / 3}^{\prime} \mathrm{M}_{1 / 3}^{\prime \prime}\right)_{2} \mathrm{CT}_{2}$.

Table II summarizes the calculated total energies of the optimized lattice structures of various iMXenes for the three different model structures functionalized with $\mathrm{F}$, $\mathrm{OH}$, and $\mathrm{O}$ groups. We find that for all iMXenes studied here, regardless of the type of functionalization, model structure $\mathcal{A} \mathcal{A}$ has the lowest energy. Accordingly, we also show the adsorption energy $E_{\mathrm{a}}$ of the functional groups on the iMXenes with the most stable model structure $\mathcal{A A}$ in Table II. These adsorption energies are large negative values, indicating the formation of strong chemical bonds between the surface and the functional groups [11]. In fact, the functional groups strengthen the stability of MXenes by saturating the nonbonding valence electrons of the transition metals [76].

We also investigate the dynamic stability of the predicted iMXene structures on the basis of a set of phonon calculations. The results for pristine and functionalized $\left(\mathrm{Mo}_{2 / 3} \mathrm{Y}_{1 / 3}\right)_{2} \mathrm{C}$ are shown in Fig. 4 as an example. The phonon results for other iMXenes are provided in the Supplemental Material [69]. The phonon frequencies of most of the studied iMXenes are positive, indicating that their structures are dynamically stable. Few of them have small negative phonon frequencies, which are generally observed in the phonon spectra of $2 \mathrm{D}$ systems and can possibly be removed by applying a small strain or deposition onto a proper substrate [77-82]. Such small negative phonon frequencies could also be an artifact of the numerical inaccuracy due to the limited supercell size, cutoff energy, or number of $k$ points, or reflect the actual lattice instability towards large wave undulations of $2 \mathrm{D}$ materials. However, $\left(\mathrm{V}_{2 / 3} \mathrm{Zr}_{1 / 3}\right)_{2} \mathrm{CO}_{2}$ shows clear negative phonon modes (see the Supplemental Material [69]), which indicates the model structure $\mathcal{A} \mathcal{A}$ of this iMXexe is not dynamically stable. Therefore, the second lowest energy structure of this iMXexe, i.e., model structure $\mathcal{A B}$, is the stable structure for $\left(\mathrm{V}_{2 / 3} \mathrm{Zr}_{1 / 3}\right)_{2} \mathrm{CO}_{2}$, which shows all positive phonon spectra.

\section{Electronic structures}

As an example of the electronic structures of iMXenes, Fig. 5 shows the results for pristine and functionalized $\left(\mathrm{Mo}_{2 / 3} \mathrm{Y}_{1 / 3}\right)_{2} \mathrm{C}$ with $\mathrm{F}$, OH, and $\mathrm{O}$. We find that the pristine $\left(\mathrm{Mo}_{2 / 3} \mathrm{Y}_{1 / 3}\right)_{2} \mathrm{C}$ iMXexe is metallic. It remains metallic upon functionalization with $\mathrm{F}$ and $\mathrm{OH}$, but it becomes semiconducting when it is functionalized with O. In more detail, as seen in Fig. 5(a), pristine $\left(\mathrm{Mo}_{2 / 3} \mathrm{Sc}_{1 / 3}\right)_{2} \mathrm{C}$ is metallic, and the states between -3 and $3 \mathrm{eV}$ are dominated mainly by the $d$ orbitals of Mo atoms. These states are formed owing to the hybridization among the $d$ orbitals of Mo atoms. The states between -6 and $-3 \mathrm{eV}$ result from the hybridization between the $p$ orbitals of $\mathrm{C}$ atoms and the $d$ orbitals of the transition metals. The $s$ orbitals of $\mathrm{C}$ atoms are hybridized with the $d$ orbitals of the transition metals in a much deeper energy region between -12 and $-10 \mathrm{eV}$.

The functionalization of pristine iMXenes with $\mathrm{F}, \mathrm{OH}$, or $\mathrm{O}$ induces hybridization between the atomic orbitals of the attached functional groups and the transition metals, and the new hybridized states appear between the $p$ and $s$ subbands of the $\mathrm{C}$ atoms. Owing to the formation of these hybridized states and the simultaneous electron transfer from the transition metals to $\mathrm{F}$ or $\mathrm{O}$ atoms, the Fermi energy shifts downwards to lower energies upon functionalization (see Fig. 5). The Fermi energy shift for the O-terminated iMXenes is larger than that for the Fterminated ones simply because of the different valencies of these two atoms.

Among the pristine and functionalized iMXenes studied here, only $\left(\mathrm{Mo}_{2 / 3} \mathrm{Y}_{1 / 3}\right)_{2} \mathrm{CO}_{2}, \quad\left(\mathrm{Mo}_{2 / 3} \mathrm{Sc}_{1 / 3}\right)_{2} \mathrm{CO}_{2}$, $\left(\mathrm{W}_{2 / 3} \mathrm{Y}_{1 / 3}\right)_{2} \mathrm{CO}_{2}$, and $\left(\mathrm{W}_{2 / 3} \mathrm{Sc}_{1 / 3}\right)_{2} \mathrm{CO}_{2}$ are semiconductors with indirect band gaps of $0.45,0.04,0.625$, and $0.675 \mathrm{eV}$, respectively. The origin of these band gaps may be attributed to the splitting of Mo $d$-orbital-dominated bands. In functionalized MXenes, the transition metals are surrounded with $\mathrm{C}$ atoms and the attached chemical groups (F, OH, or O). Such a local environment splits the $d$ bands into subbands [76]. More precisely, the states near the Fermi energy can be assigned as $t_{2 g}$ bands hybridized with $\mathrm{C}, \mathrm{F}$, or $\mathrm{O} p$ orbitals via $d p \pi$ coupling. In the case of $\left(\mathrm{Mo}_{2 / 3} \mathrm{Y}_{1 / 3}\right)_{2} \mathrm{CO}_{2},\left(\mathrm{Mo}_{2 / 3} \mathrm{Sc}_{1 / 3}\right)_{2} \mathrm{CO}_{2}$, 

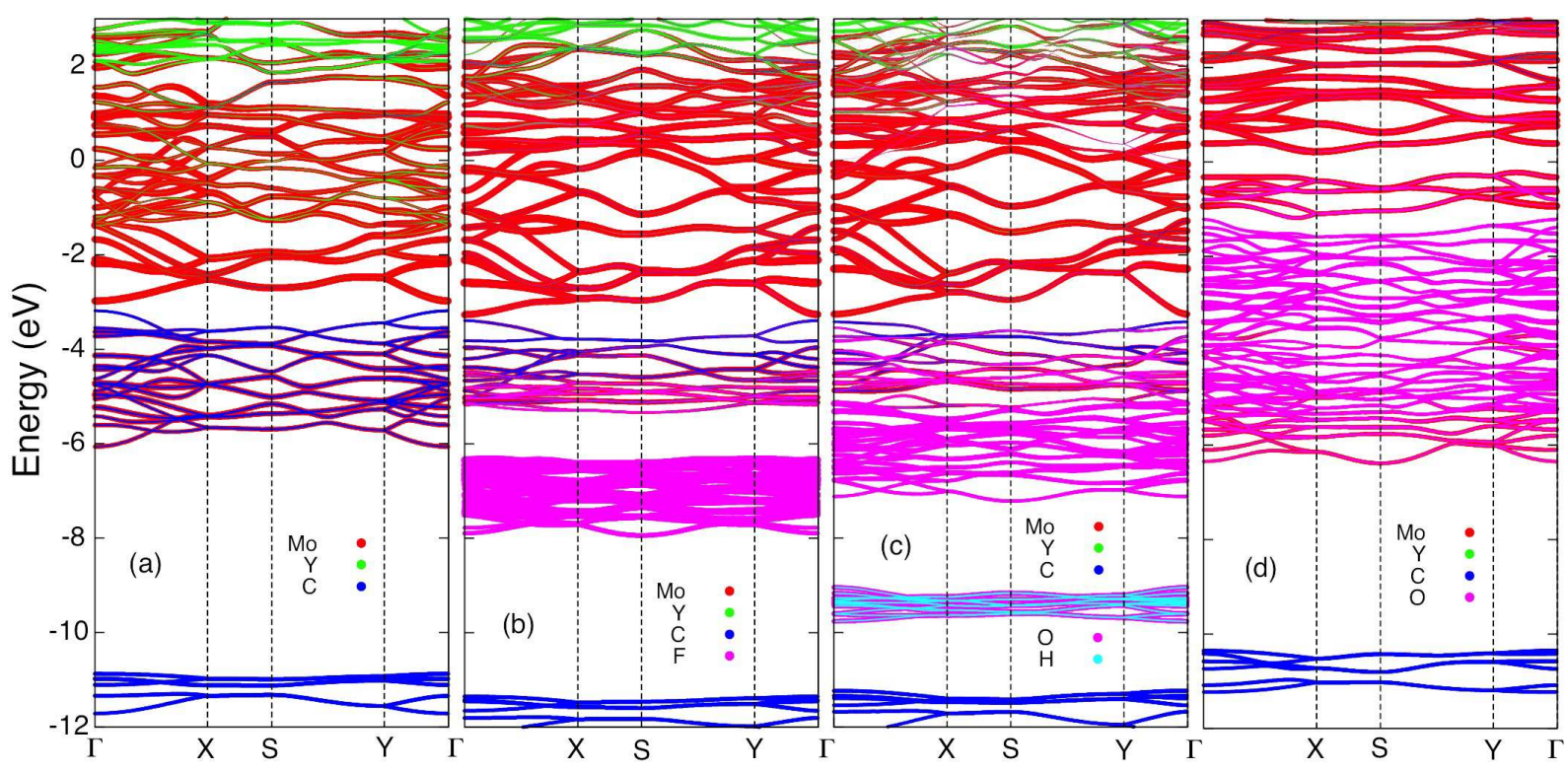

FIG. 5: Projected band structures for (a) pristine $\left(\mathrm{Mo}_{2 / 3} \mathrm{Y}_{1 / 3}\right)_{2} \mathrm{C}$, (b) $\left(\mathrm{Mo}_{2 / 3} \mathrm{Y}_{1 / 3}\right)_{2} \mathrm{CF}_{2},(\mathrm{c})\left(\mathrm{Mo}_{2 / 3} \mathrm{Y}_{1 / 3}\right)_{2} \mathrm{C}(\mathrm{OH})_{2}$, and $(\mathrm{d})$ $\left(\mathrm{Mo}_{2 / 3} \mathrm{Y}_{1 / 3}\right)_{2} \mathrm{CO}_{2}$ iMXenes. $\Gamma(0,0,0), \mathrm{X}(0.5,0,0), \mathrm{S}(0.5,0.5,0)$, and $\mathrm{Y}(0,0.5,0)$ are high symmetric points of the Brillouin zone. The Fermi energy is set at zero.

$\left(\mathrm{W}_{2 / 3} \mathrm{Y}_{1 / 3}\right)_{2} \mathrm{CO}_{2}$, and $\left(\mathrm{W}_{2 / 3} \mathrm{Sc}_{1 / 3}\right)_{2} \mathrm{CO}_{2}$, the $\mathrm{t}_{2 g}$ bands are further split by the low symmetry structure, resulting in finite band gaps. It is known that the DFT method with the GGA-PBE functional underestimates the band gaps, while hybrid functionals, e.g., Heyd-Scuseria-Ernzerhof (HSE06) [83, 84], might give a better estimate. Hence, we also examine the band gaps by using the HSE06 functional (see the Supplemental Material [69]). As expected, in the HSE06 level of theory, $\left(\mathrm{Mo}_{2 / 3} \mathrm{Sc}_{1 / 3}\right)_{2} \mathrm{CO}_{2},\left(\mathrm{Mo}_{2 / 3} \mathrm{Y}_{1 / 3}\right)_{2} \mathrm{CO}_{2}$, $\left(\mathrm{W}_{2 / 3} \mathrm{Sc}_{1 / 3}\right)_{2} \mathrm{CO}_{2}$ and $\left(\mathrm{W}_{2 / 3} \mathrm{Y}_{1 / 3}\right)_{2} \mathrm{CO}_{2}$ obtain larger band gaps of $0.58,1.23,1.3$, and $1.3 \mathrm{eV}$, respectively, than those obtained from the GGA-PBE method.

\section{Mechanical properties}

As benchmark tests for elastic property calculations, we have first considered graphene and borophene sheets [64] and found that the results obtained for $C_{11}$, $C_{22}, C_{12}$, and $C_{66}$ are in excellent agreement with those reported previously $[66,85]$. Encouraged by these results, we now calculate the elastic constants of pristine and functionalized $\left(\mathrm{Mo}_{2 / 3} \mathrm{Sc}_{1 / 3}\right)_{2} \mathrm{C},\left(\mathrm{Mo}_{2 / 3} \mathrm{Y}_{1 / 3}\right)_{2} \mathrm{C}$, $\left(\mathrm{V}_{2 / 3} \mathrm{Zr}_{1 / 3}\right)_{2} \mathrm{C}$, $\left(\mathrm{W}_{2 / 3} \mathrm{Sc}_{1 / 3}\right)_{2} \mathrm{C}$, and $\left(\mathrm{W}_{2 / 3} \mathrm{Y}_{1 / 3}\right)_{2} \mathrm{C}$ iMXenes. The results are summarized in Table III. The obtained elastic constants for all of these iMXenes satisfy the Born criteria, $C_{11}+C_{22}-C_{12}^{2}>0$ and $C_{66}>0$ [86]. This implies that all of these iMXenes are mechanically stable. Moreover, we find that all of the elastic constants of the pristine iMXenes increase with $\mathrm{F}$, $\mathrm{O}$, or $\mathrm{OH}$ functionalization, suggesting that the functionalized iMXenes have better mechanical properties than the pristine ones.
This is in good accordance with previous studies of other pristine and functionalized MXenes [87, 88].

In addition, we calculate the in-plane Young moduli $Y_{x}=\frac{1}{C_{22}}\left(C_{11} C_{22}-C_{12} C_{21}\right)$ and $Y_{y}=\frac{1}{C_{11}}\left(C_{11} C_{22}-\right.$ $C_{12} C_{21}$ ), and the Poisson's ratios $\nu_{x y}=\frac{C_{21}}{C_{22}}$ and $\nu_{y x}=$ $\frac{C_{12}}{C_{11}}$ of the iMXenes $[85,89]$, which are summarized in Table III. We find that the O-functionalized iMXenes possess the highest Young's moduli, as large as almost 200 $\mathrm{N} / \mathrm{m}$, followed by the $\mathrm{OH}$-functionalized, the F functionalized, and pristine iMXenes. For comparison, we also calculate the Young's moduli of graphene $(340 \mathrm{~N} / \mathrm{m})$ and BN $(279.2 \mathrm{~N} / \mathrm{m})$, which are in excellent agreement with previous results [90, 91]. This suggests that iMXenes, especially the pristine ones, belong to a class of soft $2 \mathrm{D}$ materials.

Interestingly, the O-functionalized iMXenes possess large Poisson's ratios, in particular, $\left(\mathrm{Mo}_{2 / 3} \mathrm{Y}_{1 / 3}\right)_{2} \mathrm{CO}_{2}$ whose Poisson's ratio can reach up to 0.57 . Note that most 2D materials have a Poisson's ratio less than 0.3 , e.g., 0.18 for graphene [92] and 0.22 for both BN [91] and $\mathrm{MoS}_{2}$ [93]. It is also noted that the Poisson's ratio of an aluminium oxide monolayer is close to 0.68 [94]. Moreover, among the studied iMXenes, pristine $\left(\mathrm{Mo}_{2 / 3} \mathrm{Y}_{1 / 3}\right)_{2} \mathrm{C}$ shows a small negative Poisson's ratio, which has only been found in a few sets of $2 \mathrm{D}$ materials [95]. Although pristine MXenes and iMXenes are difficult to realize experimentally using wet chemical exfoliation because of their strong tendency to absob $\mathrm{F}$, O, or $\mathrm{OH}$ groups, this unique property motivates experimentalists to synthesize pristine MXenes with other experimental techniques such as chemical vapor deposition. 
TABLE III: Calculated elastic constants $C_{i j}(\mathrm{~N} / \mathrm{m})$, Young's modulus $Y_{\alpha}(\mathrm{N} / \mathrm{m})$ along the $\alpha(=x$ and $y)$ direction, and Poisson's ratio $\nu_{\alpha \beta}$, the strain response in the $\alpha$ direction to the strain applied along the $\beta$ direction, for various iMXenes.

\begin{tabular}{|c|c|c|c|c|c|c|c|c|c|}
\hline iMXene & $C_{11}$ & $C_{22}$ & $C_{12}$ & $C_{66}$ & $Y_{x}$ & $Y_{y}$ & $\nu_{x y}$ & $\nu_{y x}$ & Space group \\
\hline$\overline{\left(\mathrm{Mo}_{2 / 3} \mathrm{Sc}_{1 / 3}\right)_{2} \mathrm{C}}$ & 130.944 & 121.737 & 22.220 & 39.465 & 126.888 & 117.966 & 0.183 & 0.170 & $C 2 / m$ (No. 12$)$ \\
\hline$\left(\mathrm{Mo}_{2 / 3} \mathrm{Y}_{1 / 3}\right)_{2} \mathrm{C}$ & 114.102 & 74.386 & -2.955 & 42.635 & 113.984 & 74.309 & -0.040 & -0.026 & $C 2 / m$ (No. 12$)$ \\
\hline$\left(\mathrm{W}_{2 / 3} \mathrm{Sc}_{1 / 3}\right)_{2} \mathrm{C}$ & 111.633 & 115.741 & 18.825 & 52.012 & 108.571 & 112.566 & 0.163 & 0.169 & $C 2 / m$ (No. 12$)$ \\
\hline$\left(\mathrm{W}_{2 / 3} \mathrm{Y}_{1 / 3}\right)_{2} \mathrm{C}$ & 89.747 & 113.441 & 36.990 & 45.595 & 77.685 & 98.195 & 0.326 & 0.412 & $C 2 / m$ (No. 12 ) \\
\hline$\left(\mathrm{V}_{2 / 3} \mathrm{Zr}_{1 / 3}\right)_{2} \mathrm{C}$ & 120.676 & 91.458 & 32.128 & 43.205 & 109.390 & 82.905 & 0.351 & 0.266 & $C 2 / m$ (No. 12 ) \\
\hline$\left(\mathrm{Mo}_{2 / 3} \mathrm{Sc}_{1 / 3}\right)_{2} \mathrm{CF}_{2}$ & 154.389 & 158.156 & 59.944 & 45.577 & 131.669 & 134.882 & 0.379 & 0.388 & $C 2 / m$ (No. 12 ) \\
\hline$\left(\mathrm{Mo}_{2 / 3} \mathrm{Y}_{1 / 3}\right)_{2} \mathrm{CF}_{2}$ & 136.214 & 147.712 & 54.653 & 47.224 & 115.992 & 125.783 & 0.370 & 0.401 & $C 2 / m$ (No. 12$)$ \\
\hline$\left(\mathrm{W}_{2 / 3} \mathrm{Sc}_{1 / 3}\right)_{2} \mathrm{CF}_{2}$ & 142.892 & 149.628 & 50.781 & 39.587 & 125.657 & 131.581 & 0.339 & 0.339 & $C 2 / m$ (No. 12) \\
\hline$\left(\mathrm{W}_{2 / 3} \mathrm{Y}_{1 / 3}\right)_{2} \mathrm{CF}_{2}$ & 156.307 & 155.771 & 39.698 & 45.547 & 146.189 & 145.689 & 0.255 & 0.254 & $C 2 / m$ (No. 12$)$ \\
\hline$\left(\mathrm{V}_{2 / 3} \mathrm{Zr}_{1 / 3}\right)_{2} \mathrm{CF}_{2}$ & 159.566 & 159.529 & 41.999 & 58.976 & 148.509 & 148.475 & 0.263 & 0.263 & $C 2 / m$ (No. 12 ) \\
\hline$\left(\mathrm{Mo}_{2 / 3} \mathrm{Sc}_{1 / 3}\right)_{2} \mathrm{CO}_{2}$ & 257.927 & 198.383 & 92.341 & 76.824 & 214.946 & 165.324 & 0.465 & 0.358 & $C 2$ (No. 5) \\
\hline$\left(\mathrm{Mo}_{2 / 3} \mathrm{Y}_{1 / 3}\right)_{2} \mathrm{CO}_{2}$ & 224.802 & 142.086 & 73.584 & 56.601 & 186.694 & 117.999 & 0.518 & 0.327 & $C 2($ No. 5$)$ \\
\hline$\left(\mathrm{W}_{2 / 3} \mathrm{Sc}_{1 / 3}\right)_{2} \mathrm{CO}_{2}$ & 279.897 & 219.678 & 90.389 & 85.458 & 242.705 & 190.488 & 0.411 & 0.323 & $C 2($ No. 5$)$ \\
\hline$\left(\mathrm{W}_{2 / 3} \mathrm{Y}_{1 / 3}\right)_{2} \mathrm{CO}_{2}$ & 250.670 & 173.314 & 79.150 & 68.260 & 214.524 & 148.323 & 0.457 & 0.316 & $C 2($ No. 5$)$ \\
\hline$\left(\mathrm{V}_{2 / 3} \mathrm{Zr}_{1 / 3}\right)_{2} \mathrm{CO}_{2}$ & 243.120 & 223.845 & 92.442 & 66.270 & 204.944 & 188.695 & 0.413 & 0.380 & Cm (No. 8) \\
\hline$\left(\mathrm{Mo}_{2 / 3} \mathrm{Sc}_{1 / 3}\right)_{2} \mathrm{C}(\mathrm{OH})_{2}$ & 164.041 & 164.822 & 49.382 & 54.335 & 149.246 & 149.957 & 0.300 & 0.301 & $C 2 / m$ (No. 12$)$ \\
\hline$\left(\mathrm{Mo}_{2 / 3} \mathrm{Y}_{1 / 3}\right)_{2} \mathrm{C}(\mathrm{OH})_{2}$ & 141.653 & 148.998 & 46.117 & 54.075 & 127.380 & 133.984 & 0.310 & 0.326 & $C 2 / m$ (No. 12 ) \\
\hline$\left(\mathrm{W}_{2 / 3} \mathrm{Sc}_{1 / 3}\right)_{2} \mathrm{C}(\mathrm{OH})_{2}$ & 155.277 & 153.032 & 48.042 & 54.723 & 140.195 & 138.169 & 0.314 & 0.309 & $C 2 / m$ (No. 12$)$ \\
\hline$\left(\mathrm{W}_{2} / \mathrm{Y}_{1 / 3}\right)_{2} \mathrm{C}(\mathrm{OH})_{2}$ & 143.140 & 145.001 & 36.714 & 55.714 & 133.844 & 135.584 & 0.253 & 0.256 & $C 2 / m$ (No. 12$)$ \\
\hline$\left(\mathrm{V}_{2 / 3} \mathrm{Zr}_{1 / 3}\right)_{2} \mathrm{C}(\mathrm{OH})_{2}$ & 170.367 & 159.457 & 40.705 & 63.418 & 159.976 & 149.732 & 0.255 & 0.239 & $C 2 / m$ (No. 12$)$ \\
\hline
\end{tabular}

\section{Piezoelectric properties}

Considering the symmetry of the iMXenes, indicated in Table III, the pristine, F-, and $\mathrm{OH}-$ functionalized iMXenes with $C 2 / m$ symmetry have centrosymmetry except O-functionalized ones with $C 2$ symmetry. Therefore, it can expected the semiconducting iMXenes, $\left(\mathrm{Mo}_{2 / 3} \mathrm{Y}_{1 / 3}\right)_{2} \mathrm{CO}_{2},\left(\mathrm{Mo}_{2 / 3} \mathrm{Sc}_{1 / 3}\right)_{2} \mathrm{CO}_{2}$, $\left(\mathrm{W}_{2 / 3} \mathrm{Sc}_{1 / 3}\right)_{2} \mathrm{CO}_{2}$, and $\left(\mathrm{W}_{2 / 3} \mathrm{Y}_{1 / 3}\right)_{2} \mathrm{CO}_{2}$ to have piezoelectric properties. Therefore, the two independent piezoelectric stress coefficients, $e_{11}$ and $e_{12}$, represent the piezoelectric response under the uniaxial strains $\varepsilon_{x x}$ and $\varepsilon_{y y}$. Table IV summarizes the calculated clampedand relaxed-ion piezoelectric stress coefficients [96]. The clamped ion stands for the calculations in which the positions of ions are not optimized after applying a uniaxial strain. The relaxed and clamped piezoelectric strain coefficients of $d_{11}$ and $d_{12}$, also listed in Table IV, reflect the amount of induced polarization upon applying an external force and therefore represent the mechanicalelectrical energy converting ratio. We find that the relaxed $d_{11}$ and $d_{12}$ values are notably larger for these four iMXenes than those predicted for transition metal dichalcogenides (estimated as 2.12-13.5 $\mathrm{pmV}^{-1}$ ) [9698], most monolayered materials such as BN, CaS, GaSe, GaAs, and AlSb (estimated as $0.5-3.0 \mathrm{pmV}^{-1}$ ) [68, 99101], and bulk materials including $\alpha$-quartz, wurtzite GaN, and wurtzite AlN (estimated as 2.3, 3.1, and 5.1 $\left.\mathrm{pmV}^{-1}\right)[102,103]$, which are widely used in industry.
In order to check the results above, we also calculate $e_{11}$ and $e_{12}$ from the relation $e_{i j}=\partial P_{i} / \partial \varepsilon_{j}$, where $P_{i}$ is the induced polarization evaluated by the Berry phase method along the $i$ direction in the presence of the strain $\varepsilon_{j}$ applied along the $j$ direction. The piezoelectric coefficients are obtained by a linear fitting of the change in $P_{i}$ versus $\varepsilon_{j}$, in which $\varepsilon_{j}$ varies between -0.01 and +0.01 in increments of 0.002 . We find that the obtained relaxed-ion $e_{11}$ piezoelectric coefficients of $\left(\mathrm{Mo}_{2 / 3} \mathrm{Y}_{1 / 3}\right)_{2} \mathrm{CO}_{2},\left(\mathrm{Mo}_{2 / 3} \mathrm{Sc}_{1 / 3}\right)_{2} \mathrm{CO}_{2}$, $\left(\mathrm{W}_{2 / 3} \mathrm{Sc}_{1 / 3}\right)_{2} \mathrm{CO}_{2}$, and $\left(\mathrm{W}_{2 / 3} \mathrm{Y}_{1 / 3}\right)_{2} \mathrm{CO}_{2}, 39.54,43.58$, 38.02 , and $33.89 \times 10^{-10} \mathrm{C} / \mathrm{m}$, respectively, are in excellent agreement with the DFPT results listed in Table IV. Therefore, these results clearly demonstrate that iMXenes have peculiar piezoelectric properties with $d_{11}$ one order of magnitude larger than those of commercially utilized bulk materials and most semiconducting 2D materials. Finally, we note that we have also calculated the relaxed-ion piezoelectric coefficients of $\mathrm{Sc}_{2} \mathrm{CO}_{2}$, the only system without centrosymmetry among the $\mathrm{M}_{2} \mathrm{CO}_{2}$ $(\mathrm{M}=\mathrm{Sc}, \mathrm{Ti}, \mathrm{Zr}$, and $\mathrm{Hf})$ MXenes and found that $e_{11}=3.333 \times 10^{-10} \mathrm{Cm}^{-1}$ and $d_{11}=4.137 \mathrm{pVm}^{-1}$. Therefore, we predict that the piezoelectric properties of $\mathrm{Sc}_{2} \mathrm{CO}_{2}$ are similar to those of well-known transition metal dichalcogenides, $\mathrm{MoS}_{2}$ and $\mathrm{MoSe}_{2}$ [98], but smaller than those of the iMXenes investigated in this study. 
TABLE IV: Calculated clamped- and relaxed-ion piezoelectric stress ( $e_{11}$ and $e_{12}$ in unit of $10^{-10} \mathrm{Cm}^{-1}$ ) and piezoelectric strain $\left(d_{11}\right.$ and $d_{12}$ in unit of $\left.\mathrm{pVm}^{-1}\right)$ coefficients for $\left(\mathrm{Mo}_{2 / 3} \mathrm{Sc}_{1 / 3}\right)_{2} \mathrm{CO}_{2},\left(\mathrm{Mo}_{2 / 3} \mathrm{Y}_{1 / 3}\right)_{2} \mathrm{CO}_{2},\left(\mathrm{~W}_{2 / 3} \mathrm{Sc}_{1 / 3}\right)_{2} \mathrm{CO}_{2}$, and $\left(\mathrm{W}_{2 / 3} \mathrm{Y}_{1 / 3}\right)_{2} \mathrm{CO}_{2}$ iMXenes.

\begin{tabular}{lcccc}
\hline \hline & \multicolumn{3}{c}{ Clamped-ion } & \multicolumn{2}{c}{ Relaxed-ion } \\
iMXene & $e_{11}$ & $e_{12}$ & $e_{11}$ & $e_{12}$ \\
\hline$\left(\mathrm{Mo}_{2 / 3} \mathrm{Sc}_{1 / 3}\right)_{2} \mathrm{CO}_{2}$ & 11.76 & -4.80 & 44.55 & -10.99 \\
$\left(\mathrm{Mo}_{2 / 3} \mathrm{Y}_{1 / 3}\right)_{2} \mathrm{CO}_{2}$ & 30.52 & -1.64 & 40.33 & -4.89 \\
$\left(\mathrm{~W}_{2 / 3} \mathrm{Sc}_{1 / 3}\right)_{2} \mathrm{CO}_{2}$ & 9.90 & -4.00 & 38.82 & -7.59 \\
$\left(\mathrm{~W}_{2 / 3} \mathrm{Y}_{1 / 3}\right)_{2} \mathrm{CO}_{2}$ & 8.91 & -3.36 & 35.53 & -4.84 \\
\hline & $d_{11}$ & $d_{12}$ & $d_{11}$ & $d_{12}$ \\
\hline$\left(\mathrm{Mo}_{2 / 3} \mathrm{Sc}_{1 / 3}\right)_{2} \mathrm{CO}_{2}$ & 4.63 & -3.02 & 29.24 & -14.71 \\
$\left(\mathrm{Mo}_{2 / 3} \mathrm{Y}_{1 / 3}\right)_{2} \mathrm{CO}_{2}$ & 11.44 & -4.60 & 35.91 & -13.95 \\
$\left(\mathrm{~W}_{2 / 3} \mathrm{Sc}_{1 / 3}\right)_{2} \mathrm{CO}_{2}$ & 3.65 & -2.29 & 21.67 & -9.71 \\
$\left(\mathrm{~W}_{2 / 3} \mathrm{Y}_{1 / 3}\right)_{2} \mathrm{CO}_{2}$ & 3.26 & -1.87 & 24.98 & -9.82 \\
\hline \hline
\end{tabular}

\section{CONCLUSION}

The iMAX phases stand for a group of MAX phases such as $\left(\mathrm{Mo}_{2 / 3} \mathrm{Y}_{1 / 3}\right)_{2} \mathrm{AlC},\left(\mathrm{Mo}_{2 / 3} \mathrm{Sc}_{1 / 3}\right)_{2} \mathrm{AlC}$, and $\left(\mathrm{V}_{2 / 3} \mathrm{Zr}_{1 / 3}\right)_{2} \mathrm{AlC}$ with in-plane ordered double transition metals that have recently been synthesized experimentally. Consequently, their $\mathrm{Al}$ exfoliated 2D structures are named iMXenes. On the basis of first-principles calculations, we have studied dynamic stability and electronic structures of these iMAX phases as well as the corresponding iMXenes. We have shown that all iMAX phases are metallic, while their exfoliated iMXenes can become either metallic or semiconducting upon appropriate surface functionalization. We have shown that Ofunctinalized iMXenes are semiconducting and exhibit large piezoelectric coefficients. We have also demonstrated that some iMXenes have unique mechanical properties. Because of the many interesting properties expected for iMXenes, extensive experimental studies are highly anticipated in the near future.

\section{Acknowledgments}

We would like to thank Dr. Wenbin Li for fruitful discussions. M.K. and A.R. are grateful to RIKEN Advanced Center for Computing and Communication (ACCC) for the allocation of computational resources of the RIKEN supercomputer system (HOKUSAI GreatWave). M.K. acknowledges the members of the Numerical Materials Simulator at NIMS for their continued support of the supercomputing facility. M.K. also gratefully acknowledges the support by a Grant-in-Aid for Scientific Research (No. 17K14804) from MEXT, Japan. C.S. acknowledges the support from the Scientific and Technological Research Council of Turkey (TUBITAK-116F080) and Anadolu University (BAP-1705F335). V.W. acknowledges the support by the Natural Science Basic Research Plan (Grant No. 2017JM1008) of the Shaanxi Province of China.
[1] M. W. Barsoum, The $\mathrm{M}_{N+1} \mathrm{AX}_{N}$ phases: A new class of solids: Thermodynamically stable nanolaminates, Prog. Solid State Chem. 28, 201 (2000).

[2] J. Wang and Y. Zhou, Recent progress in the theoretical prediction, preparation, and characterization of layered ternary transition-metal carbides, Annu. Rev. Mater. Res. 39, 415 (2009).

[3] Z. M. Sun, Progress in research and development on MAX Phases: a family of metallic ceramics, Int. Mater. Rev. 56, 143 (2011).

[4] D. Horlait, S. C. Middleburgh, A. Chroneos and W. E. Lee, Synthesis and DFT investigation of new bismuthcontaining MAX phases, Sci. Rep. 6, 18829 (2016).

[5] H. Fashandi, M. Dahlqvist, J. Lu, J. Palisaitis, S. I. Simak, I. A. Abrikosov, J. Rosen, L. Hultman, M. Andersson, A. L. Spetz, and P. Eklund, Synthesis of $\mathrm{Ti}_{3} \mathrm{AuC}_{2}, \mathrm{Ti}_{3} \mathrm{Au}_{2} \mathrm{C}_{2}$ and $\mathrm{Ti}_{3} \mathrm{IrC}_{2}$ by noble metal substitution reaction in $\mathrm{Ti}_{3} \mathrm{SiC}_{2}$ for high-temperature-stable Ohmic contacts to $\mathrm{SiC}$, Nat. Mater. 16, 814 (2017).

[6] M. Naguib, M. Kurtoglu, V. Presser, J. Lu, J. Niu, M. Heon, L. Hultman, Y. Gogotsi, and M. W. Barsoum, Two-dimensional nanocrystals produced by exfoliation of $\mathrm{Ti}_{3} \mathrm{AlC}_{2}$. Adv. Mater. 23, 4248 (2011).

[7] M. Naguib, O. Mashtalir, J. Carle, V. Presser, J. Lu, L. Hultman, Y. Gogotsi, and M. W. Barsoum, Twodimensional transition metal carbides, ACS Nano 6,
$1322(2012)$

[8] I. Persson, L. -Å. Näslund, J. Halim, M. W. Barsoum, V. Darakchieva, J. Palisaitis, J. Rosen, P. O. Å. Persson, 2D Mater. 5, 015002 (2017).

[9] B. Anasori, M. R. Lukatskaya, and Y. Gogotsi, 2D metal carbides and nitrides (MXenes) for energy storage, Nat. Rev. 1, 16098 (2017).

[10] M. Khazaei, A. Ranjbar, M. Arai, T. Sasaki, and Yunoki, S. Electronic properties and applications of MXenes: a theoretical review, J. Mater. Chem. C 5, 2488 (2017).

[11] M. Khazaei, M. Arai, T. Sasaki, C. -Y. Chung, N. S. Venkataramanan, M. Estili, Y. Sakka, and Y. Kawazoe, Novel electronic and magnetic properties of twodimensional transition metal carbides and nitrides, Adv. Funct. Mater. 23, 2185 (2013).

[12] M. Khazaei, M. Arai, T. Sasaki, M. Estili, and Y. Sakka, Two-dimensional molybdenum carbides: potential thermoelectric materials of the MXene family, Phys. Chem. Chem. Phys. 16, 7841 (2014).

[13] M. Khazaei, M. Arai, T. Sasaki, A. Ranjbar, Y. Liang, S. Yunoki, OH-terminated two-dimensional transition metal carbides and nitrides as ultralow work function materials, Phys. Rev. B 92, 075411 (2015).

[14] H. Weng, A. Ranjbar, Y. Liang, Z. Song, M. Khazaei, S. Yunoki, M. Arai, Y. Kawazoe, Z. Fang, and X. 
Dai, Large-gap two-dimensional topological insulator in oxygen functionalized MXene, Phys. Rev. B 92, 075436 (2015).

[15] S. Lai, J. Jeong, S. K. Jang, J. Xu, Y. J. Choi, J. -H. Park, E. Hwang and S. Lee, Nanoscale, 7, 19390 (2015).

[16] M. Khazaei, A. Ranjbar, M. Ghorbani-Asl, M. Arai, T. Sasaki, Y. Liang, and S. Yunoki, Nearly free electron states in MXenes, Phys. Rev. B 93, 205125 (2016).

[17] M. Khazaei, A. Ranjbar, M. Arai, and S. Yunoki, Topological insulators in the ordered double transition metals $\mathrm{M}_{2}^{\prime} \mathrm{M}^{\prime \prime} \mathrm{C}_{2}$ MXenes $\left(\mathrm{M}^{\prime}=\mathrm{Mo}, \mathrm{W} ; \mathrm{M}^{\prime \prime}=\mathrm{Ti}, \mathrm{Zr}, \mathrm{Hf}\right)$, Phys. Rev. B 94, 125152 (2016).

[18] F. Shahzad, M. Alhabeb, C. B. Hatter, B. Anasori, S. M. Hong, C. M. Koo, and Y. Gogotsi, Electromagnetic interference shielding with $2 \mathrm{D}$ transition metal carbides (MXenes), Science 353,1137 (2016).

[19] Y. Liang, M. Khazaei, A. Ranjbar, M. Arai, S. Yunoki, Y. Kawazoe, H. Weng, and Z. Fang, Theoretical prediction of two-dimensional functionalized MXene nitrides as topological insulators, Phys. Rev. B 96, 195414 (2017).

[20] Y. Yang, S. Umrao, S. Lai, and S. Lee, Large-area highly conductive transparent two-dimensional $\mathrm{Ti}_{2} \mathrm{CT}_{x}$ film, $\mathrm{J}$. Phys. Chem. Lett. 8, 859 (2017).

[21] C. Si, J. Zhou, and Z. Sun, Half-metallic ferromagnetism and surface functionalization-induced metal-insulator transition in graphene-like two-dimensional $\mathrm{Cr}_{2} \mathrm{C}$ crystals, ACS Appl. Mater. Interfaces 7, 17510 (2015).

[22] J. He, P. Lyu, L. Z. Sun, Á. M. García, and P. Nachtigall, High temperature spin-polarized semiconductivity with zero magnetization in two-dimensional Janus MXenes, J. Mater. Chem. C 4, 6500 (2016).

[23] M. Je, Y. Lee, and Y. -C. Chung, Structural stability and electronic properties of multi-functionalized twodimensional chromium carbides, Thin Solid Films, 619, 131 (2016).

[24] G. Gao, G. Ding, J. Li, K. Yao, M. Wu, and M. Qian, Monolayer MXenes: promising half-metals and spin gapless semiconductors, Nanoscale 8, 8986 (2016).

[25] H. Kumar, N. C. Frey, L. Dong, B. Anasori, Y. Gogotsi, and V. B. Shenoy, Tunable magnetism and transport properties in nitride MXenes, ACS Nano 11, 7648 (2017).

[26] T. Ouisse and M. W. Barsoum, Magnetotransport in the MAX phases and their 2D derivatives: MXenes, Mater. Res. Lett. 5, 365 (2017).

[27] Y. Zhang and F. Li, Robust half-metallic ferromagnetism in $\mathrm{Cr}_{3} \mathrm{C}_{2}$ MXene J. Magn. Magn. Mater 433, 222 (2017).

[28] H. Lashgari, M. R. Abolhassani, A. Boochani, S. M. Elahi, and J. Khodadadi, Electronic and optical properties of 2D graphene-like compounds titanium carbides and nitrides: DFT calculations, Solid State Commun. 195, 61 (2014).

[29] A. Chandrasekaran, A. Mishra, and A. K. Singh, Ferroelectricity, antiferroelectricity, and ultrathin 2D electron/hole gas in multifunctional monolayer MXene, Nano Lett. 17, 3290 (2017).

[30] F. Zhang, Z. Zhang, H. Wang, C. H. Chan, N. Y. Chan, X. X. Chen, and J. -Y. Dai, Plasma-enhanced pulsedlaser deposition of single-crystallineMo2C ultrathin superconducting, Phys. Rev. Mater., 1, 034002 (2017).

[31] J. -J. Zhang and S. Dong, J. Chem. Phys. 146, 034705
(2017).

[32] Y. Xie, Y. Dall'Agnese, M. Naguib, Y. Gogotsi, M. W. Barsoum, H. L. Zhuang, and P. R. C. Kent, Prediction and characterization of MXene nanosheet anodes for non-lithium-ion batteries, ACS Nano 8, 9606 (2014).

[33] R. B. Rakhi, B. Ahmed, M. N. Hedhili, D. H. Anjum, and H. N. Alshareef, Effect of postetch annealing gas composition on the structural and electrochemical properties of $\mathrm{Ti}_{2} \mathrm{CT}_{x}$ MXene electrodes for supercapacitor applications, Chem. Mater. 27, 5314 (2015).

[34] H. Zhang, Z. Fu, R. Zhang, Q. Zhang, H. Tian, D. Legut, T. C. Germann, Y. Guo, S. Du, and J. S. Francisco, Designing flexible 2D transition metal carbides with strain-controllable lithium storage, Proc. Natl. Acad. Sci. U.S.A., doi: 10.1073/pnas.1717219115.

[35] H. Lin, X. Wang, L. Yu, Y. Chen, and J. Shi, Twodimensional ultrathin MXene ceramic nanosheets for photothermal conversion, Nano Lett. 17, 384 (2017).

[36] H. Kim, B. Anasori, Y. Gogotsi, and H. N. Alshareef, Thermoelectric properties of two-dimensional molybdenum-based MXenes, Chem. Mater. 29, 6472 (2017).

[37] G. Fan, X. Li, Y. Ma, Y. Zhang, J. Wu, B. Xu, T. Sun, D. Gao, and J. Bi, Magnetic, recyclable $\mathrm{Pt}_{y} \mathrm{Co}_{1-y} / \mathrm{Ti}_{3} \mathrm{C}_{2} \mathrm{X}_{2}(\mathrm{X}=\mathrm{O}, \mathrm{F})$ catalyst: a facile synthesis and enhanced catalytic activity for hydrogen generation from the hydrolysis of ammonia borane, New J. Chem. 41, 2793 (2017).

[38] J. Ran, G. Gao, F. -T. Li, T. -Y. Ma, A. Du, and S. -Z. Qiao, $\mathrm{Ti}_{3} \mathrm{C}_{2}$ MXene co-catalyst on metal sulfide photoabsorbers for enhanced visible-light photocatalytic hydrogen production, Nat. Commun. 8, 13907 (2017).

[39] X. Zhang, J. Xu, H. Wang, J. Zhang, H. Yan, B. Pan, J. Zhou, and Y. Xie, Ultrathin nanosheets of MAX phases with enhanced thermal and mechanical properties in polymeric compositions: $\mathrm{Ti}_{3} \mathrm{Si}_{0.75} \mathrm{Al}_{0.25} \mathrm{C}_{2}$, Angew. Chem. Int. Ed. Engl. 52, 4361 (2013).

[40] M. Xue, Z. Wang, F. Yuan, X. Zhang, W. Wei, H. Tang, and C. Li, Preparation of $\mathrm{TiO}_{2} / \mathrm{Ti}_{3} \mathrm{C}_{2} \mathrm{~T}_{x}$ hybrid nanocomposites and their tribological properties as base oil lubricant additives, RSC Adv. 7, 4312 (2017).

[41] Z. Sun, R. Ahuja, and J. M. Schneider, Theoretical investigation of the solubility in $\left(\mathrm{M}_{x} \mathrm{M}_{2-x}^{\prime}\right) \mathrm{AlC}$ ( $\mathrm{M}$ and $\mathrm{M}^{\prime}=\mathrm{Ti}, \mathrm{V}, \mathrm{Cr}$ ), Phys. Rev. B 68, 224112 (2003).

[42] M. F. Cover, O. Warschkow, M. M. M. Bilek, and D. R. McKenzie, A comprehensive survey of $\mathrm{M}_{2} \mathrm{AX}$ phase elastic properties, J. Phys.: Condens. Matter 21, 305403 (2009).

[43] M. Dahlqvist, B. Alling, and J. Rosén, Stability trends of MAX phases from first principles, Phys. Rev. B 81, 220102(R) (2010).

[44] Y. Mo, P. Rulis, and W. Y. Ching, Electronic structure and optical conductivities of 20 MAX-phase compounds, Phys. Rev. B 86, 165122 (2012).

[45] S. Aryal, R. Sakidja, M. W. Barsoum, and W. -Y. Ching, A genomic approach to the stability, elastic, and electronic properties of the MAX phases, Phys. Satus Solidi B 251, 1480 (2014).

[46] M. Khazaei, M. Arai, T. Sasaki, M. Estili, amd Y. Sakka, Trends in electronic structures and structural properties of MAX phases: a first-principles study on $\mathrm{M}_{2} \mathrm{AlC}\left(\mathrm{M}=\mathrm{Sc}, \mathrm{Ti}, \mathrm{Cr}, \mathrm{Zr}, \mathrm{Nb}, \mathrm{Mo}, \mathrm{Hf}\right.$, or Ta), $\mathrm{M}_{2} \mathrm{AlN}$, and hypothetical $\mathrm{M}_{2} \mathrm{AlB}$ phases, J. Phys.: Condens. Matter 26, 505503 (2014). 
[47] M. Khazaei, M. Arai, T. Sasaki, M. Estili, and Y. Sakka, The effect of the interlayer element on the exfoliation of layered $\mathrm{Mo}_{2} \mathrm{AC}(\mathrm{A}=\mathrm{Al}, \mathrm{Si}, \mathrm{P}, \mathrm{Ga}, \mathrm{Ge}, \mathrm{As}$ or In $)$ MAX phases into two-dimensional $\mathrm{Mo}_{2} \mathrm{C}$ nanosheets, Sci. Tech. Adv. Mater. 15, 014208 (2014).

[48] M. Dahlqvist and J. Rosén, Order and disorder in quaternary atomic laminates from first-principles calculations, Phys. Chem. Chem. Phys. 17, 31810 (2015).

[49] M. Ashton, R. G. Hennig, S. R. Broderick, K. Rajan, and S. B. Sinnott, Computational discovery of stable $\mathrm{M}_{2} \mathrm{AX}$ phases, Phys. Rev. B 94, 054116 (2016).

[50] A. Talapatra, T. Duong, W. Son, H. Gao, M. Radovic, and R. Arróyave, High-throughput combinatorial study of the effect of $\mathrm{M}$ site alloying on the solid solution behavior of $\mathrm{M}_{2} \mathrm{AlC}$ MAX phases, Phys. Rev. B 94, 104106 (2016).

[51] R. Arróyave, A. Talapatra, T. Duong, W. Son, H. Gao, and M. Radovic, Does aluminum play with well others? Intrinsic Al-Al alloying behavior in 211/312 MAX phases, Mater. Res. Lett. 5, 170 (2017).

[52] B. Anasori, M. Dahlqvist, J. Halim, E. J. Moon, J. Lu, B. C. Hosler, E. N. Caspi, S. J. May, L. Hultman, P. Eklund, J. Rosén and M. W. Barsoum, Experimental and theoretical characterization of ordered MAX phases $\mathrm{Mo}_{2} \mathrm{TiAlC}_{2}$ and $\mathrm{Mo}_{2} \mathrm{Ti}_{2} \mathrm{AlC}_{3}$, J. Appl. Phys. 118, 94304 (2015).

[53] R. Meshkian, Q. Tao, M. Dahlqvist, J. Lu, L. Hultman and J. Rosen, Theoretical stability and materials synthesis of a chemically ordered MAX phase, $\mathrm{Mo}_{2} \mathrm{ScAlC}_{2}$, and its two-dimensional derivate $\mathrm{Mo}_{2} \mathrm{ScC}_{2}$ MXene, Acta Mater. 125, 476 (2017).

[54] Q. Tao, M. Dahlqvist, J. Lu, S. Kota, R. Meshkian, J. Halim, J. Palisaitis, L. Hultman, M. W. Barsoum, P. O. Persson and J. Rosen, Two-dimensional Mo1.33C MXene with divacancy ordering prepared from parent $3 \mathrm{D}$ laminate with in-plane chemical ordering, Nat. Commun. 8, 14949 (2017).

[55] M. Dahlqvist, J. Lu, R. Meshkian, Q. Tao, L. Hultman and J. Rosen, Prediction and synthesis of a family of atomic laminate phases with Kagomé-like and in-plane chemical ordering, Sci. Adv. 3, e1700642 (2017).

[56] R. Meshkian, M. Dahlqvist, J. Lu, B. Wickman, J. Halim, J. Thörnberg, Q. Tao, S. Li, S. Intikhab, J. Snyder, M. W. Barsoum, M. Yildizhan, J. Paiisaitis, L. Hultman, P. O. A. Persson, J. Rosen, W-based atomic laminates and their $2 \mathrm{D}$ derivative $\mathrm{W}_{1.33} \mathrm{C} \mathrm{MX}-$ ene with vacancy ordering, Adv. Mater. (2018). DOI: 10.1002/adma.201706409.

[57] E. N. Caspi, P. Chartier, F. Porcher, F. Damay and T. Cabioc, Ordering of $(\mathrm{Cr}, \mathrm{V})$ Layers in Nanolamellar $\left(\mathrm{C}_{0.5} \mathrm{~V}_{0.5}\right)_{n+1} \mathrm{AlC}_{n}$ Compounds, Mater. Res. Lett. 3100 (2015).

[58] T. L. Tan, H. M. Jin, M. B. Sullivan, B. Anasori and Y. Gogotsi, High-throughput survey of ordering configurations in MXene alloys across compositions and temperatures, ACS Nano, 11, 4407 (2017).

[59] A. Thore and J. Rosen, An investigation of the in-plane chemically ordered atomic laminates $\left(\mathrm{Mo}_{2 / 3} \mathrm{Sc}_{1 / 3}\right)_{2} \mathrm{AlC}$ and $\left(\mathrm{Mo}_{2 / 3} \mathrm{Y}_{1 / 3}\right)_{2} \mathrm{AlC}$ from first principles, Phys. Chem. Chem. Phys. 19, 21595 (2017).

[60] G. Kresse and J. Furthmüller, Efficiency of ab-initio total energy calculations for metals and semiconductors using a plane-wave basis set, Comput. Mater. Sci. 6, 15 (1996).
[61] J. P. Perdew, K. Burke, and M. Ernzerhof, Generalized gradient approximation made simple, Phys. Rev. Lett. 77, 3865 (1996).

[62] H. J. Monkhorst and J. D. Pack, Special points for Brillouin-zone integrations, Phys. Rev. B 13, 5188 (1976).

[63] A. Togo, F. Oba, and I. Tanaka, First-principles calculations of the ferroelastic transition between rutile-type and $\mathrm{CaCl}_{2}$-type $\mathrm{SiO}_{2}$ at high pressures, Phys. Rev. B 78, 134106 (2008).

[64] V. Wang and W.T. Geng, Lattice defects and the mechanical anisotropy of borophene, J. Phys. Chem. C 121, 10224 (2017).

[65] R. C. Andrew, R. E. Mapasha, A. M. Ukpong, and N. Chetty, Mechanical properties of graphene and boronitrene, Phys. Rev. B 85, 125428 (2012).

[66] S. Q. Wang and H. Q. Ye, Ab initio elastic constants for the lonsdaleite phases of $\mathrm{C}, \mathrm{Si}$ and Ge, J. Phys.: Condens. Matter 15, 5307 (2003).

[67] R. W. Nunes and X. Gonze, Berry-phase treatment of the homogeneous electric field perturbation in insulators, Phys. Rev. B 63, 155107 (2001).

[68] R. Fei, W. Li, J. Li, and L. Yang, Giant piezoelectricity of monolayer group IV monochalcogenides: SnSe, SnS, GeSe, and GeS, Appl. Phys. Lett. 107, 173104 (2015).

[69] See Supplemental Material at http://link.aps.org/supplemental/10.1103/ for the phonon dispersions and HSE06 band structure calculations.

[70] M. Khazaei, A. Ranjbar, K. Esfarjani, B. Dimitri, R. Dronskowski, and S. Yunoki, Insights into exfoliation possibility of MAX phases to MXenes, Phys. Chem. Chem. Phys. 20, 8579 (2018).

[71] H. Lind, J. Halim, S. I. Simak and J. Rosen, Investigation of vacancy-ordered $\mathrm{Mo}_{1} .33 \mathrm{C}$ MXene from first principles and x-ray photoelectron spectroscopy, Phys. Rev. Mater. 1, 044002 (2017).

[72] M. Ashton, K. Mathew, R. G. Hennig and S. B. Sinnott, Predicted surface composition and thermodynamic stability of MXenes in solution, J. Phys. Chem. C 120, 3550 (2016).

[73] L. Li, Lattice dynamics and electronic structures of $\mathrm{Ti}_{3} \mathrm{C}_{2} \mathrm{O}_{2}$ and $\mathrm{Mo}_{2} \mathrm{TiC}_{2} \mathrm{O}_{2}$ (MXenes): The effect of Mo substitution, Comput. Mater. Sci. 124, 8 (2016).

[74] C. Si, K. -H. Jin, J. Zhou, Z. Sun and F. Liu, Large-gap quantum spin Hall state in MXenes: $d$-band topological order in a triangular lattice, Nano Lett. 16, 6584 (2016).

[75] C. Si, J. You, W. Shi, J. Zhou, and Z. Sun, Quantum spin Hall phase in $\mathrm{Mo}_{2} \mathrm{M}_{2} \mathrm{C}_{3} \mathrm{O}_{2}(\mathrm{M}=\mathrm{Ti}, \mathrm{Zr}$, Hf) MXenes, J. Mater. Chem. C 4, 11524 (2016).

[76] T. Hu, Z. Li, M. Hu, J. Wang, Q. Hu, Q. Li and $\mathrm{X}$. Wang, Chemical origin of termination-functionalized MXenes: $\mathrm{Ti}_{3} \mathrm{C}_{2} \mathrm{~T}_{2}$ as a case study, J. Phys. Chem. C 121, 19254 (2017).

[77] X. Li, Y. Dai, M. Li, W. Wei and B. Huang, Stable Si-based pentagonal monolayers: high carrier mobilities and applications in photocatalytic water splitting, J. Mater. Chem. A 3, 24055 (2015).

[78] E. S. Penev, A. Kutana, and B. I. Yakobson, Can TwoDimensional Boron Superconduct? Nano Lett. 16, 2522 (2016).

[79] W. Sun, Y. Li, B. Wang, X. Jiang, M. I. Katsnelson, P. Korzhavyi, O. Erikssona, and I. D. Marco, A new 2D monolayer BiXene, $\mathrm{M}_{2} \mathrm{C}(\mathrm{M}=\mathrm{Mo}$, Tc, Os) Nanoscale 8, 
15753 (2016)

[80] J. Lei, A. Kutana, and B. I. Yakobson, Predicting stable phase monolayer $\mathrm{Mo}_{2} \mathrm{C}$ (MXene), a superconductor with chemically-tunable critical temperature, J. Mater. Chem. C 5, 3438 (2017).

[81] J. He, X. Li, P. Lyu and P. Nachtigall, Near-roomtemperature Chern insulator and Dirac spin-gapless semiconductor: nickel chloride monolayer, Nanoscale $\mathbf{9}$, 2246 (2017).

[82] M. Mushtaq, Y. Zhou and X. Xiang, $\mathrm{NiX}_{2}(\mathrm{X}=\mathrm{Cl}$ and $\mathrm{Br})$ sheets as promising spin materials: a first-principles study, RSC Adv. 7, 22541 (2017).

[83] J. Heyd, G. E. Scuseria, and M. Ernzerhof, Hybrid functionals based on a screened Coulomb potential, J. Chem. Phys. 118, 8207 (2003).

[84] J. Heyd, G. E. Scuseria, and M. Ernzerhof, Erratum: "Hybrid functionals based on a screened Coulomb potential" [J. Chem. Phys. 118, 8207 (2003)], J. Chem. Phys. 124, 219906 (2006).

[85] A. J. Mannix, X. -F. Zhou, B. Kiraly, J. D. Wood, D. Alducin, B. D. Myers, X. Liu, B. L. Fisher, U. Santiago, J. R. Guest, M. J. Yacaman, A. Ponce, A. R. Oganov, M. C. Hersam and N. P. Guisinger, Synthesis of borophenes: Anisotropic, two-dimensional boron polymorphs, Science 350, 1513 (2015).

[86] M. Born and H. Huang, Dynamical Theory of Crystal Lattices (Clarendon, Oxford, 1954).

[87] Z. Guo, J. Zhou, C. Sia and Z. Sun, Flexible twodimensional $\mathrm{Ti}_{n+1} \mathrm{C}_{n}$ ( $n=1,2$ and 3$)$ and their functionalized MXenes predicted by density functional theories, Phys. Chem. Chem. Phys. 17, 15348 (2015).

[88] Z. H. Fu, Q. F. Zhang, D. Legut, C. Si, T. C. Germann, T. Lookman, S. Y. Du, J. S. Francisco, and R. F. Zhang, Stabilization and strengthening effects of functional groups in two-dimensional titanium carbide, Phys. Rev. B 94, 104103 (2016).

[89] Q. Wei and X. Peng, Superior mechanical flexibility of phosphorene and few-layer black phosphorus, Appl. Phys. Lett. 104, 251915 (2014).

[90] C. Lee, X. Wei, J. W. Kysar and J. Hone, Measurement of the elastic properties and intrinsic strength of monolayer graphene, Science 321, 385 (2008).
[91] Q. Peng, W. Ji and S. De, Mechanical properties of the hexagonal boron nitride monolayer: ab initio study, Comput. Mater. Sci. 56, 11 (2012).

[92] F. Liu, P. Ming, and J. Li, Ab initio calculation of ideal strength and phonon instability of graphene under tension, Phys. Rev. B 76, 064120 (2007).

[93] J. L. J. Feldman, Elastic constants of $2 \mathrm{H}-\mathrm{MoS} 2$ and $2 \mathrm{H}-$ $\mathrm{NbSe} 2$ extracted from measured dispersion curves and linear compressibilities, Phys. Chem. Solids 37, 1141 (1976).

[94] T. T. Song, M. Yang, J. W. Chai, M. Callsen, J. Zhou, T. Yang, Z. Zhang, J. S. Pan, D. Z. Chi, Y. P. Feng and S. J. Wang, The stability of aluminium oxide monolayer and its interface with two-dimensional materials, Sci. Rep. 6, 29221 (2016).

[95] Z. Gao, X. Dong, N. Li, and J. Ren, Novel twodimensional silicon dioxide with in-plane negative Poisson's ratio, Nano Lett. 17, 772 (2017).

[96] M. M. Alyörük and Y. Aierken and D. Çakir and F. M. Peeters and C. Sevik, Promising piezoelectric performance of single Layer transition-metal dichalcogenides and dioxides, J. Phys. Chem. C 119, 23231 (2015).

[97] K. A. N. Duerloo and M. T. Ong, and E. J. Reed, J. Phys. Chem. Lett. 3, 2871 (2012).

[98] M. N. Blonsky, H. L. Zhuang, A. K. Singh, and R. G. Hennig, Ab initio prediction of piezoelectricity in twodimensional materials, ACS Nano 9, 9885 (2015).

[99] W. Li and J. Li, Piezoelectricity in two-dimenional group-III monochalcogenides, Nano Res. 8, 3796 (2015).

[100] H. Zheng, X. -B. Li, N. -K. Chen, Sheng-Yi Xie, Wei Quan Tian, Yuanping Chen, Hong Xia, S. B. Zhang, and Hong-Bo Sun, Monolayer II-VI semiconductors: A firstprinciples prediction, Phys. Rev. B 92, 115307 (2015).

[101] C. Sevik, D. Çakir, O. Glseren, and F. M. Peeters, Peculiar Piezoelectric Properties of Soft Two-Dimensional Materials, J. Phys. Chem. C 120, 13948 (2016).

[102] R. Behmann, Elastic and piezoelectric constants of alpha-quartz, Phys. Rev. 110, 1060 (1958).

[103] C. M. Lueng, H. L. W. Chan, C. Surya, and C. L. Choy, Piezoelectric coefficient of aluminum nitride and gallium nitride, J. Appl. Phys. 88, 5360 (2000). 